Volumen 16 Número 3, Julio - Septiembre 2021, pp. 1-22, e671

THE ECONOMICS AND FINANCE EFFECTS OF THE COVID-19 PANDEMIC Editor: Dr. Ignacio Perrotini

DOI: https://doi.org/10.21919/remef.v16i3.671

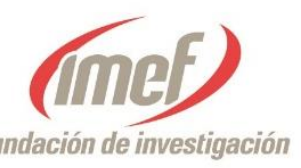

(Recibido: 25/enero/2021, aceptado: 30/abril/2021, publicado: 3/junio/2021)

\title{
La pandemia Covid-19, la crisis financiera y la dinámica (Overshooting) del tipo de cambio
}

\author{
Heri Oscar Landa Díaz' ${ }^{1}$ - Universidad Autónoma Metropolitana, México \\ Verónica Cerezo García ${ }^{2}$ - Universidad Nacional Autónoma de México, México
}

Nuestro objetivo es analizar la dinámica del tipo de cambio ante las políticas expansivas adoptadas por varios países para confrontar choques exógenos durante el periodo 2000:01-2020:03. Nuestros resultados, fundamentados en un modelo ARDL y funciones de impulso-respuesta, confirman la presencia de overshooting en Corea, México, Reino Unido y Estados Unidos. En Alemania, Japón y Brasil no encontramos evidencia de overshooting, probablemente debido al carácter semifijo del euro, la reprimarización de la economía brasileña y el estancamiento con deflación de Japón. Nuestra contribución original y conclusión más importante consiste en que las políticas expansivas para paliar la crisis financiera de 2007/08 y la inducida por el Covid-19 afectan la dinámica del tipo de cambio, lo cual implica efectos significativos en: la deuda externa, la hoja de balance de los sectores institucionales, la balanza de pagos y el crecimiento económico. Es recomendable que los gobiernos adopten medidas para contrapesar estos desequilibrios. Las limitaciones de nuestro estudio estriban en que, por ausencia de suficientes datos, no estimamos la magnitud del impacto del overshooting inducido por la pandemia en estas últimas variables.

Clasificación JEL: I18, G10, F31, F37, F4.

Palabras clave: Covid-19, crisis financiera, tipo de cambio, overshooting.

\section{The Covid-19 Pandemic, the Financial crisis and the Dynamic (Overshooting) of the Exchange Rate}

The aim of this paper is to analyze the dynamics of the exchange rate (E) in the face of expansionary policies undertaken to cope with external shocks over the period 2000: 01-2020: 03. Our results, based on an ARDL model and impulse-response functions, confirm evidence of overshooting of $E$ in South Korea, Mexico, the United Kingdom and the United States, while in Germany, Brazil and Japan no such evidence was found; perhaps this is due to the semi-fixed nature of the euro, the reprimarization of the Brazilian trade structure and Japan's stagnation cum deflation. Our original contribution and main conclusion is that expansionary policies undertaken to tame the effects of both the 2007/08 financial crisis and the Covid-19 pandemic act upon the dynamics of E, in turn implying significant effects on: foreign debt, institutional sectors' balance sheets, balance of payments and economic growth. Hence governments should adopt countervailing measures, it is highly recommended. Lack of sufficient data to estimate the impact of E overshooting, triggered by the Covid19 , on the latter variables is the main shortcoming of the paper.

JEL Classification: I18, G10, F31, F37, F41.

Keywords: Covid-19, financial crisis, exchange rate, overshooting.

${ }^{1}$ Autor de correspondencia. Universidad Autónoma Metropolitana Unidad Iztapalapa. Correo electrónico: hold77@hotmail.com

2 Escuela Nacional de Estudios Superiores Unidad León de la Universidad Nacional Autónoma de México (UNAM), Correo electrónico: vcgcerezo@yahoo.com.

*Sin fuente de financiamiento para el desarrollo de la investigación 


\begin{abstract}
And now, in the present Covid-19 crisis, there has never been more clamour from economists and policy-makers for government action to support aggregate demand. We are all Keynesians now (except, perhaps, for Robert Lucas)!

Anthony P. Thirlwall (220, p. 354).
\end{abstract}

\title{
1. Introducción
}

La pandemia Covid-19 ha perpetrado un enorme impacto en la economía mundial. Los estragos globales de la pandemia en la economía superan a los que infringió la gran crisis financiera de 20072008 y se parangonan con la Gran Depresión de 1929-1933. El Fondo Monetario Internacional (FMI, World Economic Outlook, 2021) estima que la contracción de la economía global en 2020 será de -3.5 \% y proyecta una recuperación global de 5.5\% en 2021 y de $4.2 \%$ en 2022. Pese a este repunte, en los próximos años se registrarán brechas de producto negativas y elevadas tasas de desempleo en todo el mundo.

Por ejemplo, el producto interno bruto (PIB) de Estados Unidos cayó -4.3\% en 2020 (la primera contracción desde 2009), la tasa de desempleo aumentó a 14.8\% en abril de ese año -la cifra más alta desde la Gran Depresión de 1929- y aún en enero de 2021 se mantuvo en 6.3\%, muy superior al nivel previo a la irrupción de la pandemia (3.5\%). Incluso las economías más dinámicas de Asia también experimentaron una fuerte desaceleración, el crecimiento económico en China descendió de $6.1 \%$ a $1.9 \%$ entre 2019 y 2020 , el de Corea del Sur de $2 \%$ a $-1.9 \%$ en el mismo periodo debido principalmente a la disminución del consumo y de las exportaciones. La economía mexicana se contrajo $-9 \%$ en 2020.

Con el propósito de detener el contagio los gobiernos de todos los países decidieron cerrar de forma abrupta las actividades productivas, sociales, escolares y hasta las fronteras para controlar la propagación del virus. Estas acciones de lockdown han infligido un enorme costo en todos los sectores económicos y en los hogares, cuya magnitud varía según la fortaleza o debilidad relativa en que se encontraba la estructura económica de cada nación al momento de la irrupción del virus. Para paliar los efectos negativos de la pandemia Covid-19, los gobiernos y los bancos centrales aplicaron políticas monetarias no convencionales y de estímulo fiscal, mediante la implementación de programas de expansión del gasto público y de la liquidez, disminución de las tasas de interés, adquisición de activos, facilidades de crédito para reactivar a las empresas en el corto plazo, moratorias de pago de deudas y de impuestos, transferencias directas a desempleados y hogares con restricción de liquidez (Aguilar y Cantú, 2020; BIS, 2020; Board of Governors of the Federal Reserve System, 2020).

El objetivo del presente artículo es probar empíricamente si, en un contexto de prolongada estabilidad de precios, el fenómeno de overshooting ocurre cuando la economía confronta una crisis (como la financiera de 2007-2008 o la asociada al Covid-19) y la autoridad monetaria y el gobierno realizan políticas monetarias y fiscales expansivas para combatir la recesión. La principal contribución de este trabajo es aportar evidencia del fenómeno de overshooting o sobreajuste del tipo de cambio en un grupo de países como consecuencia de los efectos de la pandemia Covid-19 en 
la economía y de la política monetaria y fiscal expansiva adoptada para paliar la contracción de la actividad económica. Además, el periodo de estudio comprende las dos principales crisis económicas del siglo XXI, lo cual nos permite comparar lo ocurrido en ambos momentos críticos, 2007-2008 y 2020.

El artículo está organizado de la siguiente forma. A continuación, presentamos el argumento esencial de la teoría del overshooting del tipo de cambio, en la tercera parte damos cuenta de manera sucinta de la literatura empírica acerca de algunas experiencias de sobrerreacción del tipo de cambio. La cuarta contiene el análisis empírico y la última es la conclusión.

\section{Dinámica del tipo de cambio: el fenómeno de overshooting}

Como consecuencia de la crisis y ulterior cancelación del sistema monetario y financiero internacional de Bretton Woods a principios de la década de los años 1970, varios países adoptaron regímenes de tipo de cambio flexible. Un fenómeno observado en los primeros años desde la adopción de la libre flotación monetaria fue la sobrerreacción o sobreajuste en el corto plazo de los tipos de cambio ante choques monetarios o de demanda exógenos. Este sobreajuste contrastaba con la menor y más lenta reacción del producto, de los precios y de la inflación medida por el índice de precios al consumidor (Dornbusch, 1976; Bhandari, 1982). La mayor velocidad de reacción de los tipos de cambio, relativa a los precios, en el corto plazo, implicaba también una diferente velocidad de retorno al valor de equilibrio o de largo plazo de los tipos de cambio, de las tasas de interés y de los precios.

El fenómeno antes descrito fue analizado de forma pionera por Dornbusch (1976) en un contexto de alta inflación y crecientes tasas de desempleo que caracterizaron a la crisis de estanflación (estancamiento con inflación) de la década de los años setenta. A esto Dornbusch lo denominó overshooting del tipo de cambio.

El abandono de regímenes de tipo de cambio fijos (y ajustables), característico de Bretton Woods, tuvo dos implicaciones principales. Por una parte, la paridad de tipo de cambio nominal dejó de ser el ancla de la inflación; por otra, la libre flotación del tipo de cambio dio lugar a fluctuaciones abruptas de las paridades cambiarias ante choques exógenos de oferta o de demanda. Estas fluctuaciones abruptas dieron lugar a reacciones excesivas de corto plazo de las monedas.

La primera implicación antes mencionada significó que la política monetaria deviniera en el principal mecanismo regulador de los precios. En el caso de Estados Unidos este cambio estructural comenzó en 1979 con la llamada desinflación Volcker (Hetzel, 2008). Volcker puso el énfasis en las expectativas de las negociaciones salariales de los sindicatos, las políticas de precios de las empresas y las decisiones financieras de las corporaciones que "han estimulado el proceso inflacionario" (Volcker, 1980:9). La estrategia monetaria de Volcker consistió entonces en restringir el crecimiento de la oferta monetaria, con lo cual se redujo el aumento de los precios y se agudizaron las condiciones para el sobreajuste del tipo de cambio del dólar en caso de un choque exógeno.

El experimento Volcker fue abandonado en 1982 y fue sustituido por un marco de política monetaria en el que la tasa de interés de los fondos federales reemplazó a los agregados monetarios como instrumento para el control de la inflación. Así, esta nueva estrategia monetaria de la Reserva Federal originó un prolongado periodo, 1982-2007, de tasas de inflación baja y estable conocido como la Gran Moderación. Europa. Asia y América Latina adoptaron la misma estrategia de control 
de la inflación a partir de la década de los años 1990. Con el advenimiento de la Gran Crisis Financiera de 2007-2008, la Reserva Federal abandonó este modelo y adoptó una política monetaria no convencional en la que la hoja de balance del banco central es el instrumento principal (véase Perrotini, 2015).

La segunda implicación es más relevante para nuestro análisis presente. Desde el experimento Volcker los precios de los bienes que conforman el PIB se han mantenido rígidos al alza, tanto en Estados Unidos como en el resto de la economía mundial, excepto en algunos países como Argentina y Venezuela, entre otros, cuya inestabilidad obedece a factores idiosincrásicos; sin embargo, las tasas de interés, los precios de los activos financieros y el tipo de tipo de cambio de las diversas monedas han tenido un comportamiento más volátil. Es decir, el éxito obtenido en el control de la inflación no ha evitado que, sobre todo en momentos de crisis económica, se verifiqué el fenómeno de overshooting del tipo de cambio. La crisis financiera de 2007-2008 provocó sobreajustes del tipo de cambio impulsados por choques monetarios exógenos. Y ahora la crisis asociada al doble choque de oferta y demanda que representa el efecto económico de la pandemia Covid-19 ha dado lugar a sobrerreacciones del tipo de cambio mayores que la reacción del nivel de precios.

¿Qué factores explican esta volatilidad del tipo de cambio? La teoría económica establece que existe una relación entre el tipo de cambio nominal (E), las tasas de interés nacional (i) e internacional ( $\mathrm{i}^{*}$ ) y el mercado monetario (donde la oferta monetaria es $\mathrm{M}$ y la demanda de dinero es $\mathrm{P}_{0} Y_{0} \mathrm{~L}_{\mathrm{i}} ; Y$ es el producto y $\mathrm{L}$ es la demanda de liquidez; en equilibrio $\mathrm{M}=\mathrm{P}_{0} Y_{0} \mathrm{~L}_{\mathrm{i}}$ ). El enfoque monetario del tipo de cambio postula que el precio relativo de los activos financieros nacionales y los activos financieros internacionales está determinado por la paridad de tasas de interés:

$$
i=i *+\left(E^{e}-E\right) / E
$$

Donde $E^{e}$ denota las expectativas del tipo de cambio. La paridad de tasas de interés descubierta supone que el riesgo cambiario es cero, los inversores son neutrales al riesgo. Suponiendo perfecta sustitución entre ambos activos financieros, las diferencias entre sus tasas rendimientos es eliminada por los movimientos del tipo de cambio; de este modo se equilibran las tasas de rendimiento y el tipo de cambio es el precio relativo de los dos activos financieros:

$$
E=E^{e} /(1+i-i *)
$$

Las expectativas de tipo de cambio dependen de la paridad de poder de compra (P/P*). Dado que, de acuerdo con la teoría cuantitativa del dinero, un choque monetario provocará un aumento en los precios, las expectativas de tipo de cambio de largo plazo estarán determinadas por los movimientos de largo plazo de $\left(p_{l p} / p^{*}{ }_{l p}\right)$ :

$$
E^{e}=\alpha\left(p_{l p} / p_{l p}^{*}\right)
$$

Donde $\alpha \leq 1$ es el coeficiente que mide la velocidad de ajuste del tipo de cambio respecto de los movimientos de los precios relativos inducidos por el choque monetario. Ante un desequilibrio 
monetario $\left(\mathrm{M}>\mathrm{P}_{0} Y_{0} \mathrm{~L}_{\mathrm{i}}\right)$, supongamos que como resultado de una expansión monetaria realizada por el banco central para atenuar los efectos de un choque de oferta y de demanda como el que representa la pandemia Covid-19, se suscitará un doble efecto: primero, de acuerdo con la paridad de tasas de interés, el exceso de dinero en circulación provocará un descenso inmediato de la tasa de interés $i$ y una devaluación del tipo de cambio de corto plazo. Segundo, con base en la paridad de poder de compra, el exceso monetario provocará, en el largo plazo, un aumento en el nivel de precios $p$ y una depreciación de la moneda nacional.

Lo que significa este modelo es que, dado un choque monetario, el ajuste del tipo de cambio de corto plazo determinado por la paridad de tasas de interés excede al ajuste del tipo de cambio de largo plazo determinado por la paridad de poder de compra. Esto sucede porque ante un choque monetario exógeno, la velocidad de ajuste de los mercados financieros es mayor que la del mercado de bienes. La causa es que las tasas de interés y el tipo de cambio son más flexibles que los precios. Esta dinámica del tipo de cambio es lo que Dornbusch (1976) identificó como el fenómeno de overshooting. En el supuesto de expectativas racionales, la depreciación del tipo de cambio será menor en la nueva posición de equilibrio que en el caso de expectativas estáticas, lo que significa una apreciación respecto a este último caso. Y dado que los agentes racionales anticipan correctamente esta apreciación, la tasa de interés interna o nacional será menor que con expectativas estáticas.

El tipo de cambio también puede experimentar un sobreajuste como resultado de ataques especulativos monetarios, eventos que suelen dar lugar a efectos de contagio. Dornbusch et al. (2000) distinguen entre "shift contagion" y "pure contagion". El primero ocurre cuando el choque que experimenta un país se transmite a otros países con los que sostiene vínculos comerciales y/o financieros, mientras que el segundo se caracteriza porque no se relaciona con cambios en los fundamentales de la economía, sus causas pueden ser conductas irracionales de rebaño o efectos riqueza derivados de pérdidas de capital en los mercados financieros. Los mecanismos de transmisión del contagio son varios, desde el comercio internacional, los flujos financieros a través de la banca internacional, los reajustes en los portafolios de inversión y los cambios en la información de los mercados financieros y en la política macroeconómica internacional.

\section{Breve revisión de la literatura empírica}

Tomando pie en un modelo de vectores autorregresivos estructural (SVAR), Kim et al. (2020) analizan el efecto de los choques en política monetaria sobre las fluctuaciones del tipo de cambio, en siete economías de Asia durante el periodo de 2000:01 a 2018:03. En general, sus estimaciones confirman que las perturbaciones de la tasa de interés local (aumento) inducen un proceso de sobrerreacción del tipo de cambio (apreciación) en Corea del Sur, Malasia y China, mientras que los resultados sugieren una depreciación cambiaria en India e Indonesia. En Tailandia y Filipinas las estimaciones muestran que el tipo de cambio no reacciona de manera significativa a una contracción de la política monetaria. Asimismo, encuentran que, con excepción del peso filipino, la prima de riesgo tiene un impacto relevante en la dinámica de la tasa cambiaria, tanto en el corto como en el largo plazo.

En un estudio para Estados Unidos, Rüth (2020) analiza si una contracción inesperada de la política monetaria provoca un sobreajuste (apreciación) del tipo de cambio, antes de depreciarse gradualmente, durante el periodo 1979:07-2012:06. En sus regresiones, realizadas mediante un 
modelo SVAR, identifica un proceso de sobrerreacción del dólar ante una perturbación de la política monetaria del Banco de la Reserva Federal, condición que sustenta la hipótesis de Dornbusch (1976). Asimismo, sus resultados revelan que, ante un cambio exógeno de la tasa de interés de los fondos federales (aumento), la producción y los precios declinan hasta alcanzar una senda uniforme en el horizonte del pronóstico, al tiempo que confirman que la prima de riesgo constituye un instrumento efectivo (mecanismo de aceleración financiera) para absorber choques monetarios.

En un trabajo sobre México, Capistrán et al. (2019) analizan la hipótesis de sobrerreacción del tipo de cambio de Dornbusch, durante el periodo 1990:01-2015:04. En sus regresiones, elaboradas con base en un modelo ARDL y un sistema de vectores autorregresivos con corrección de error estructural (SVEC), hallan una relación de largo plazo entre el tipo de cambio, la tasa de interés y la variación de precios, mientras que los resultados sugieren la persistencia de un efecto negativo en la paridad cambiaria ante variaciones de la demanda de dinero y el producto. También, encuentran causalidad bidireccional entre la tasa de interés y el tipo de cambio, lo que confirma que el banco central reacciona endógenamente a los choques cambiarios para amortiguar cualquier proceso de traspaso inflacionario.

Ghosh y Bhadury (2017) analizan, mediante un modelo de vectores autorregresivos (VAR), la validez empírica de la hipótesis de sobrerreacción del tipo de cambio de Dornbusch en los casos de Australia, Nueva Zelanda, Suecia y Canadá durante el periodo 1987:01-2004:04. En sus resultados encuentran que el impacto del choque monetario en la oferta o la demanda genera una depreciación del tipo de cambio en el corto plazo y, posteriormente, una apreciación en el largo plazo que apoya la validez de la hipótesis de sobrerreacción del tipo de cambio y la condición de paridad de interés no cubierta.

Güneş y Karul (2016) estudian la consistencia de la hipótesis de overshooting en Turquía con datos mensuales correspondientes al período 2000-2014. En sus regresiones, realizadas mediante un modelo autorregresivo con rezagos distribuidos (ADRL), hallan evidencia que respalda la hipótesis de sobreajuste del tipo de cambio aun cuando los coeficientes no son estadísticamente significativos para todas las variables, excepto en el caso de la respuesta del tipo de cambio a la tasa de interés en el corto y largo plazo. Según sus estimaciones, la expansión monetaria induce una apreciación de la lira turca, de esta forma un incremento en la tasa de interés provoca una sobrerreacción en el tipo de cambio en el corto y largo plazo.

Renani, et al. (2014) analizan la validez de la hipótesis de overshooting en Irán durante el período 2001-2010, con base en un modelo de vectores autorregresivos con corrección de error (VEC). Sus estimaciones incluyen datos de Irán y Estados Unidos sobre oferta monetaria, tipo de cambio, producto interno bruto real y tasa de inflación, en las que encuentran evidencia de que un choque monetario provoca overshooting en el tipo de cambio en el corto y largo plazo.

\section{Análisis empírico}

\subsection{Hechos estilizados}

El presidente de Estados Unidos, Joe Biden, firmó recientemente un paquete de estímulo por la cantidad de 1.9 trillones de dólares, equivalente a 9\% del PIB. Se espera que esto incremente el consumo -que representa más de dos tercios de la demanda agregada de ese país-, la Oficina de 
Presupuesto del Congreso estima un incremento igual al 5\% del PIB y que esto reactive al mercado de trabajo y a la economía en general (Congressional Budget Office, 2021). La Reserva Federal (Fed) pronostica que la economía estadounidense crecerá 6.5\% en 2021, 3.3\% en 2022, 2.2\% en 2023 y la tasa de desempleo será de 4.5\%. Es decir, el paquete de estímulos reactivará el PIB sólo en el corto plazo.

El gobierno de China, a su vez, incrementó el gasto público, emitió 1.2 trillones de yuanes en bonos de deuda pública; el banco central de China disminuyó la tasa de reserva de requerimiento con lo cual liberó 500 billones de yuanes de fondos en el largo plazo. El gobierno de Japón también aprobó en abril 2020 un programa de emergencia económica anti Covid-19 por la suma de 108 trillones de yenes; el "Fondo de Apoyo Amplio de Emergencia para las Infecciones del Coronavirus" destinado a fortalecer el sistema de salud asciende a 149 billones de yenes (Niki, 2020). Además, en el tercer presupuesto suplementario aprobado en enero 2021, el gobierno japonés aprobó 4.4 trillones de yenes para contener la propagación del Covid-19. De igual manera, el gobierno de la República de Corea puso por práctica políticas de lockdown, fiscales y monetarias similares: entre febrero y julio de 2020 suministró liquidez en moneda nacional, Won, en cantidad igual a 217 trillones, la mayor parte del estímulo se destinó al rescate (bailout) de las mega corporaciones financieras y no financieras: $60 \%$ a las principales corporaciones industriales de exportación y financieras; $26 \%$ a las pequeñas y medianas industrias; $7 \%$ a los hogares en la forma de incentivos fiscales y transferencias de ingresos de emergencia; $1 \%$ para estabilizar el empleo y 1.2\% para financiar la salud pública. El gobierno coreano ha logrado estimular el consumo con estas medidas. En el caso de México, también el gobierno implementó programas de gasto público orientados a apoyar a los sectores sociales más vulnerables (mediante transferencias) pero sin abandonar su enfoque de finanzas públicas sanas: en 2020, a pesar de la recesión y de la menor recaudación tributaria, se registró un superávit primario equivalente a $0.7 \%$ del PIB, con lo cual el gobierno federal satisface la meta anual de $0.1 \%$ que ha caracterizado a la política fiscal en años recientes. Acaso el hecho de que México no utilizó su espacio fiscal disponible, dada la baja razón deuda/PIB del país, contribuya a explicar la severidad de la recesión Covid-19 de 2020. El Banco de México, asimismo, realizó un conjunto de acciones de relajamiento monetario, entre las que están: reducción de la tasa de interés a $6 \%$ y ajuste de la tasa de interés ordinaria de la liquidez adicional a 1.1 veces la tasa de política monetaria; una línea swap con la Fed por 60 mil millones de dólares; reducción de los depósitos de regulación monetaria; aumentos en la liquidez de los mercados financieros y de los sistemas de pagos, de coberturas de divisas y operaciones de crédito en dólares; intercambio de valores de deuda por títulos del gobierno; recompra de valores corporativos; financiamiento a bancos comerciales y de desarrollo, en algunos casos utilizando como colateral a corporaciones emisoras de deuda pública; coberturas de divisas y swaps de valores gubernamentales LT y facilidades para fortalecer el Programa Market Makers para valores gubernamentales.

Todas estas políticas orientadas a mitigar los efectos del Covid-19 en la economía, en la disminución del ingreso de las empresas y los hogares debido al aislamiento y a la pausa de la actividad productiva, por una parte, son bienvenidas; por otra, implican una extraordinaria presión financiera para el sector público y para el sector privado. El estímulo fiscal y monetario en muchos casos aumentará el servicio de la deuda pública. Estos son tópicos que han sido discutidos con cierta amplitud. Sin embargo, hasta ahora ha estado ausente el análisis del impacto de las políticas expansivas, fiscal y monetaria, adoptadas ante la crisis sanitaria, en los tipos de cambio de las 
diversas monedas. La cuestión es si estas políticas han inducido un efecto de sobrerreacción monetaria. Dada la relevancia del tipo de cambio en una economía abierta (en la actualidad todas las economías son abiertas, de ahí el rápido del Covid-19 a escala global) y dado el agudo problema del llamado "pecado original" (con excepción de Estados Unidos, los gobiernos y corporaciones tienden a endeudarse en monedas extranjeras, principalmente en dólares, fenómeno conocido como "currency mistmatch"), es pertinente examinar si las intervenciones fiscales y monetarias han dado lugar al fenómeno denominado overshooting del tipo de cambio. La importancia de una devaluación de esta naturaleza no se puede soslayar, puesto que la depreciación monetaria devalúa los activos de los deudores, incrementa la deuda externa de las empresas que operan en el sector de bienes no comerciables (el mercado interno), incrementa el costo de la deuda y afecta la hoja de balance de los sectores institucionales de la economía.

Si bien durante los últimos veinte años las tasas de interés han mantenido un comportamiento sincronizado con el objetivo de control de la inflación, la crisis financiera de 20072008 y los choques de oferta y demanda derivados de la crisis sanitaria Covid-19, cuyos efectos más crudos se han puesto de manifiesto en 2020, han inducido una reducción significativa de las tasas de interés que las ha abatido hasta el límite cero. Estas crisis también han provocado que los bancos centrales -especialmente los de los países desarrollados (véanse cuadros 1 y 2)- aumenten de forma sustancial la oferta monetaria y que los gobiernos abandonen su austeridad y expandan su política de gasto público.

La orientación de estas medidas para confrontar el choque exógeno en la economía provocado por la pandemia Covid-19 ha tenido un carácter expansivo para proveer liquidez al mercado de dinero/activos, amortiguar la desaceleración de la demanda agregada y, por ende, la del producto. Sin embargo, con temporalidades y profundidades distintas para cada país, estas políticas anti-recesivas también imponen presiones significativas en la formación de precios, las expectativas de inflación, la toma de riesgos en el sector financiero (apalancamiento y rentabilidad) y en la variabilidad de los tipos de cambio.

Cuadro 1. Tipo cambio, mercado de dinero y actividad económica

\begin{tabular}{|c|c|c|c|c|c|c|c|c|c|c|c|c|c|c|}
\hline \multirow[b]{2}{*}{ Indicador } & \multicolumn{2}{|c|}{ México } & \multicolumn{2}{|c|}{$\begin{array}{l}\text { Corea del } \\
\text { Sur }\end{array}$} & \multicolumn{2}{|c|}{ Brasil } & \multicolumn{2}{|c|}{ Reino Unido } & \multicolumn{2}{|c|}{ Alemania } & \multicolumn{2}{|c|}{ Japón } & \multicolumn{2}{|c|}{$\begin{array}{l}\text { Estados } \\
\text { Unidos }\end{array}$} \\
\hline & $\begin{array}{c}2000 \\
- \\
2010\end{array}$ & $\begin{array}{c}2010 \\
- \\
2019\end{array}$ & $\begin{array}{c}2000 \\
- \\
2010\end{array}$ & $\begin{array}{c}2010 \\
- \\
2019\end{array}$ & $\begin{array}{c}2000 \\
- \\
2010\end{array}$ & $\begin{array}{c}2010 \\
- \\
2019\end{array}$ & $\begin{array}{c}2000 \\
- \\
2010\end{array}$ & $\begin{array}{c}2010 \\
- \\
2019\end{array}$ & $\begin{array}{c}2000 \\
- \\
2010\end{array}$ & $\begin{array}{c}2010 \\
- \\
2019\end{array}$ & $\begin{array}{c}2000 \\
- \\
2010\end{array}$ & $\begin{array}{c}2010 \\
- \\
2019\end{array}$ & $\begin{array}{c}2000 \\
- \\
2010\end{array}$ & $\begin{array}{c}2010 \\
- \\
2019\end{array}$ \\
\hline $\begin{array}{l}\text { Tipo de } \\
\text { cambio } / 1\end{array}$ & 2.9 & 4.8 & 0.2 & 0.1 & -0.4 & 9.4 & -0.2 & 2.1 & -3.6 & 1.9 & -2.0 & 2.4 & -2.6 & 1.9 \\
\hline $\begin{array}{ll}\text { Tasa } & \text { de } \\
\text { interés } / 2 & \end{array}$ & 8.3 & 5.0 & 3.9 & 2.1 & 15.7 & 9.9 & 4.2 & 0.6 & 4.0 & 1.1 & 0.1 & 0.0 & 2.5 & 0.6 \\
\hline Inflación /1 & 4.7 & 3.9 & 3.2 & 1.6 & 6.6 & 5.9 & 2.1 & 2.0 & 1.6 & 1.4 & -0.3 & 0.6 & 2.4 & 1.8 \\
\hline $\begin{array}{l}\text { Oferta } \\
\text { monetaria } / 1\end{array}$ & 7.6 & 7.5 & 4.9 & 7.5 & 8.4 & -0.8 & 7.5 & 3.5 & 7.1 & 5.7 & 5.8 & 4.9 & 2.2 & 7.3 \\
\hline $\mathrm{PIB} / 1$ & 1.5 & 2.4 & 4.7 & 2.9 & 3.7 & 0.8 & 1.6 & 1.8 & 0.8 & 1.7 & 0.6 & 0.9 & 1.7 & 2.3 \\
\hline Deuda /3 & 40.7 & 49.3 & 23.7 & 37.9 & 68.0 & 72.0 & 44.3 & 83.9 & 65.8 & 72.5 & $\begin{array}{c}172 . \\
4\end{array}$ & $\begin{array}{c}230 . \\
3\end{array}$ & 91.2 & $\begin{array}{c}134 . \\
1\end{array}$ \\
\hline
\end{tabular}




\begin{tabular}{|l|c|c|c|c|c|c|c|c|c|c|c|c|c|c|}
\hline $\begin{array}{l}\text { Balance fiscal } \\
\text { /3 }\end{array}$ & -2.2 & -3.2 & 1.5 & 1.5 & -3.4 & -5.8 & -3.6 & -5.0 & -2.5 & 0.2 & -6.6 & -5.8 & -5.8 & -7.1 \\
\hline $\begin{array}{l}\text { Reservas } \\
\text { internacional } \\
\text { es /1 }\end{array}$ & 10.3 & 2.9 & 9.1 & 2.0 & 21.3 & 0.6 & 4.3 & 4.7 & 6.9 & -1.4 & 9.2 & 0.2 & 11.6 & -1.1 \\
\hline $\begin{array}{l}\text { Capitalizació } \\
\mathrm{n} \text { bursátil /3 }\end{array}$ & 26.4 & 36.6 & 63.1 & 88.6 & 53.1 & 47.1 & $\begin{array}{c}120 . \\
4\end{array}$ & $\begin{array}{c}117 . \\
5\end{array}$ & 46.2 & 47.3 & 72.2 & 92.4 & $\begin{array}{c}122 . \\
5\end{array}$ & $\begin{array}{c}135 . \\
6\end{array}$ \\
\hline
\end{tabular}

Fuente: Elaboración de los autores con datos de International Financial Statistics, Banco de la Reserva Federal, Banco Mundial y la Organización para la Cooperación y el Desarrollo Económicos.

1/ Tasa de crecimiento promedio anual; 2/ indicador como promedio anual simple; 3/ Indicador como proporción del PIB.

Aunque transversalmente persiste el principio de balance estructural y, con ello, la sincronización de la política fiscal con las metas de inflación, fundamentos del nuevo consenso macroeconómico, entre 2000 y 2019, con excepción de Corea del Sur y Alemania, la deuda y el déficit fiscal mantienen una trayectoria expansiva, condición que constituye un factor restrictivo relevante, ya que su profundización generará coacciones sobre la viabilidad futura de la inversión privada, el margen del gasto público, la capacidad de la autoridad monetaria para proveer liquidez y la calidad de los bonos gubernamentales (riesgo país), particularmente en las economías emergentes. Esto podría dar lugar a un ajuste general configurado por un proceso de histéresis del crecimiento económico (estancamiento económico con estabilidad inflacionaria) y un deslizamiento controlado del tipo de cambio (depreciación).

Otra arista de los hechos estilizados yace en las reservas internacionales, dado su papel como instrumento de compensación ante choques externos, cuya acumulación después de 2010 advierte una visible desaceleración, entre otros factores, derivado de la normalización de la política monetaria en Estados Unidos, la relocalización de capitales, las variaciones en la valuación de activos y la intervención cambiaria, especialmente en el caso de los mercados emergentes.

En efecto, después de la crisis de hipotecas subprime de 2007-2008, las buenas perspectivas sobre las ganancias corporativas y la tendencia a la baja de las tasas de interés de corto plazo, en algunos países, permitieron un rápido aumento del valor del mercado bursátil. Sin embargo, este escenario vislumbra conatos de volatilidad como la sobrevaloración de los mercados accionarios y la disociación de éstos con la dinámica del resto de la actividad económica, debido a las potenciales presiones que los movimientos no anticipados en los mercados de capital podrían generar sobre las expectativas cambiarias, la rentabilidad financiera y, por ende, la liquidez/crédito de mediano y largo plazo.

Cuadro 2. Mercados de dinero y cambiario: choques externos

\begin{tabular}{|l|c|c|c|c|c|c|c|c|c|c|c|c|c|c|}
\hline & \multicolumn{2}{|c|}{ México } & \multicolumn{2}{c|}{ Corea del Sur } & \multicolumn{2}{c|}{ Brasil } & \multicolumn{2}{c|}{$\begin{array}{c}\text { Reino } \\
\text { Unido }\end{array}$} & \multicolumn{2}{c|}{ Alemania } & \multicolumn{2}{c|}{ Japón } & \multicolumn{2}{c|}{$\begin{array}{c}\text { Estados } \\
\text { Unidos }\end{array}$} \\
\hline & $\begin{array}{c}2008- \\
2009\end{array}$ & $\begin{array}{c}2019- \\
2020\end{array}$ & $\begin{array}{c}2008- \\
2009\end{array}$ & $\begin{array}{c}2019- \\
2020\end{array}$ & $\begin{array}{c}2008- \\
2009\end{array}$ & $\begin{array}{c}2019- \\
2020\end{array}$ & $\begin{array}{c}2008- \\
2009\end{array}$ & $\begin{array}{c}2019- \\
2020\end{array}$ & $\begin{array}{c}2008- \\
2009\end{array}$ & $\begin{array}{c}2019- \\
2020\end{array}$ & $\begin{array}{c}2008- \\
2009\end{array}$ & $\begin{array}{c}2019- \\
2020\end{array}$ & $\begin{array}{c}2008- \\
2009\end{array}$ & $\begin{array}{c}2019- \\
2020\end{array}$ \\
\hline $\begin{array}{l}\text { Tipo de de } \\
\text { cambio/1 }\end{array}$ & 21.1 & 13.0 & 15.8 & 3.3 & 9.0 & 30.7 & 17.5 & 0.2 & 5.2 & 0.0 & -9.5 & -1.5 & 4.6 & 0.3 \\
\hline $\begin{array}{l}\text { Tasa de } \\
\text { interés /2 }\end{array}$ & 5.5 & 5.7 & 2.0 & 0.8 & 10.1 & 3.1 & 1.2 & 0.4 & 3.2 & -0.5 & 0.1 & -0.1 & 0.2 & 0.5 \\
\hline
\end{tabular}


REMEF (The Mexican Journal of Economics and Finance)

La pandemia Covid-19, la crisis financiera y la dinámica (Overshooting) del tipo de cambio

\begin{tabular}{|l|c|c|c|c|c|c|c|c|c|c|c|c|c|c|}
\hline $\begin{array}{l}\text { Inflación } \\
/ 1\end{array}$ & 5.3 & 3.4 & 2.8 & 0.6 & 4.9 & 2.9 & 2.0 & 1.1 & 0.3 & 0.8 & -1.4 & 0.3 & -0.4 & 1.2 \\
\hline $\begin{array}{l}\text { Oferta } \\
\text { monetaria } \\
/ 1\end{array}$ & 7.2 & -3.2 & 13.7 & 18.9 & 2.5 & 26.6 & 0.5 & 11.0 & 10.3 & 10.6 & 1.9 & 9.6 & 14.5 & 25.5 \\
\hline PIB /1 & -5.1 & -9.8 & 0.8 & -0.8 & 0.2 & -5.4 & -4.1 & -11.0 & -5.6 & -5.8 & -5.7 & -6.0 & -2.5 & -3.9 \\
\hline Deuda /3 & 43.7 & 65.5 & 30.0 & 48.4 & 65.5 & 101.4 & 63.3 & 108.0 & 73.0 & 73.3 & 200.9 & 266.2 & 115.7 & $\begin{array}{c}131 . \\
2\end{array}$ \\
\hline $\begin{array}{l}\text { Balance } \\
\text { fiscal /3 }\end{array}$ & -4.1 & -5.8 & -1.3 & -3.2 & -3.2 & -16.8 & -10.1 & -16.5 & -3.2 & -8.2 & -10.2 & -14.2 & -13.1 & -18.7 \\
\hline
\end{tabular}

Fuente: Elaboración de los autores con datos del International Financial Statistics, Banco de la Reserva Federal y la Organización para la Cooperación y el Desarrollo Económicos.

1/ Tasa de crecimiento promedio anual; 2/ indicador como promedio anual simple; 3/ Indicador como proporción del PIB.

Ciertamente, la profunda contracción del PIB, dada la desaceleración de la demanda agregada, constituye uno de los principales efectos adversos de la pandemia del Covid-19, ya que este escenario viene sincronizado con un decremento relevante de la tasa de empleo y un proceso de reconcentración de la distribución del ingreso, cuya simbiosis erosiona significativamente las expectativas y condiciones socioeconómicas de los hogares. Hay que acotar que los efectos del "paro administrado" de la actividad económica (estrategia de contención de la pandemia) han sido totalmente asimétricos, en algunos sectores económicos los desbalances requerirán de la instrumentación de políticas focalizadas, especialmente en países semi-industrializados como México, para evitar un mayor deterioro; tal es el caso de los servicios relacionados con la actividad turística, el transporte, el alojamiento y el esparcimiento. La volatilidad económica y financiera, así como la recuperación misma, estarán supeditadas a las características estructurales en materia de inversión/ahorro, productividad y desarrollo tecnológico, consistente con el impacto diferenciado que en este rubro se observa.

El choque financiero y económico propagado por la crisis sanitaria indujo fuertes correcciones cambiarias (sobrerreacción del tipo de cambio), entre otros factores, generadas por la turbulencia de los mercados bursátiles, la alta movilidad de capitales, la reducción de las tasas de interés, la expansión monetaria y fiscal, así como por la contracción de los precios de materias primas.

\subsection{Aspectos metodológicos}

La comprobación de la hipótesis de Dornbusch de overshooting o sobrerreacción del tipo de cambio ante choques externos la efectuamos en dos etapas: primero, la relación de largo plazo se aproxima mediante una especificación ARDL; segundo, con base en el procedimiento de Johansen-Joselius, obtenemos funciones de impulso-respuesta para simular los efectos de una perturbación de corto plazo sobre el comportamiento cambiario y de precios. En la primera parte del análisis, la función de reacción del tipo de cambio de largo plazo se estima mediante la siguiente especificación dinámica: 


$$
\Delta \ln e_{t}=\Gamma_{i} \ln e_{t-1}+\beta_{i}^{\prime} \tau_{t}+\sum_{j=1}^{p-1} \phi_{i j}^{*} \Delta \ln e_{t-j}+\sum_{j=0}^{q-1} \theta_{i j}^{*} \Delta \tau_{t-j}+u_{t}+\varepsilon_{t}
$$

Donde $e$ y $\tau$ constituyen, respectivamente, el tipo de cambio nominal y un vector de $k x 1$ variables explicativas (tasa de interés local y foránea, inflación, demanda de dinero, producto interno bruto); el parámetro $\Gamma_{i}$ captura la velocidad de ajuste del desequilibrio ${ }^{3}$, mientras que $\beta_{i}^{\prime}$ agrupa los parámetros de largo y los vectores $\phi_{i j}$ y $\theta_{\mathrm{ij}}^{\prime}$ los estimadores de corto plazo, en tanto que $u_{t}$ y $\varepsilon_{t}$ capturan los efectos fijos y el termino de error, correspondientemente.

Empíricamente nos interesa comprobar si el tipo de cambio nominal experimenta un desajuste significativo (sobrerreacción) respecto de su nivel de largo plazo ante un cambio no anticipado de la oferta monetaria, donde la variación de los precios internos es el mecanismo de ajuste que permite a la paridad cambiaria retornar a su senda de equilibrio (Dornbusch, 1976).

En virtud de la conjetura anterior, se espera que un aumento en la cantidad de dinero (M1), derivado de un choque externo de oferta y/o demanda, genere un desplazamiento transitorio y atípico (depreciación/apreciación) del tipo de cambio $\left(e_{t}\right)$, así como de los precios al consumidor $\left(\pi_{t}\right)$.

El uso de la metodología ARDL (véanse Blackburne y Frank, 2007; Pesaran et al, 1999)4, en contraste con otros métodos de estimación, exhibe las siguientes ventajas: i) la formulación incorpora, a través de un mecanismo de corrección de error, las desviaciones respecto de la senda de equilibrio a la dinámica de largo plazo; ii) permite capturar la heterogeneidad no observable; en consecuencia, los estimadores de corto plazo, la varianza de los errores y el coeficiente de cointegración pueden suponerse heterogéneos entre los grupos y los parámetros de pendiente de largo plazo homogéneos para cada corte transversal; iii) aún en muestras pequeñas se producen estimaciones eficientes y consistentes, controlando por correlación serial y heteroscedasticidad.

\subsection{Interpretación de resultados}

El estudio recoge información trimestral para el periodo 2000:01-2020:03 de Alemania, Brasil, Corea del Sur, Estados Unidos, Japón, México y Reino Unido. El panel incluye datos sobre producto interno bruto (PIB), tipo de cambio nominal, índice de precios al consumidor, agregado monetario M1, tasa de interés local y foránea (títulos del tesoro de Estados Unidos, tipo interbancario de oferta de Londres y bonos del gobierno de la zona euro); los cuales se encuentran en los repositorios estadísticos de la OCDE y el Fondo Monetario Internacional.

El procedimiento empírico inicia con el estudio de las propiedades estocásticas de las series, mediante pruebas de raíz unitaria de Dickey-Fuller Aumentada (ADF) y Phillips-Perron (PP), cuyos resultados sugieren que las variables son integradas I(1) en niveles y procesos estocásticos

\footnotetext{
${ }^{3} \mathrm{Si} \Gamma_{i}=0$, no existe evidencia de una relación de largo plazo. Se espera que $\Gamma_{i}<0$, bajo el supuesto de que las variables muestran un retorno hacia un equilibrio de largo plazo.

${ }^{4}$ Como en el caso del método de momentos generalizados (GMM), ya que, en contexto de muestras con $\mathrm{N}$ pequeña $\mathrm{y}$ $\mathrm{T}$ grande, produce sesgos significativos, pues en la medida que aumenta el número de variables endógenas lo hacen también los instrumentos, especialmente cuando la unidad de tiempo crece, generando una sobre-identificación del modelo y, con ello, una limitada robustez de la matriz de varianza-covarianza.
} 
estacionarios, $I(0)$, en primeras diferencias. Posteriormente, verificamos si los componentes de la función de reacción del tipo de cambio conservan co-movimiento de largo plazo siguiendo el procedimiento de Pesaran, et al (2001); confirmamos la presencia de un co-movimiento de largo plazo en las regresiones (véase anexo).

En general, según se observa en los cuadros (3) y (4), las estimaciones confirman que las variaciones de la oferta monetaria promueven choques temporales (depreciación) sobre el tipo de cambio en Corea del Sur, México, Reino Unido y Estados Unidos; en estos resultados, el deslizamiento cambiario es más profundo en el caso del peso mexicano, la libra esterlina y el dólar americano, entre otros aspectos, por la disímil velocidad de ajuste entre el mercado de bienes y el de activos, el volumen de negociación de estas divisas en los mercados internacionales, la especulación financiera, las presiones fiscales y de deuda.

Si bien la fuerte inyección de liquidez de los Bancos Centrales durante la inestabilidad y las crisis económica/financiera de 2008 y 2020 se orientó a amortiguar los efectos generados por la burbuja inmobiliaria y la emergencia sanitaria por Covid-19, respectivamente, también ha establecido una fase de volatilidad cambiaria en los principales mercados financieros, especialmente en economías emergentes, debido a la dúctil sensibilidad de la demanda de dinero respecto a la tasa de interés y a la velocidad de ajuste de las expectativas.

Cuadro 3. Sobrerreacción del tipo de cambio y choques externos

\begin{tabular}{|c|c|c|c|c|c|c|c|}
\hline Variable & $M E X$ & $M E X$ & $K O R$ & $K O R$ & $B R A$ & $B R A$ & $U K^{1 /}$ \\
\hline \multirow{2}{*}{ Constante } & 16.2437 & 13.9368 & 4.7816 & 2.9199 & 45.1208 & 33.3096 & -23.3917 \\
& {$[0.000]$} & {$[0.005]$} & {$[0.002]$} & {$[0.265]$} & {$[0.001]$} & {$[0.002]$} & {$[0.051]$} \\
\hline \multirow{2}{*}{ TID } & 2.9416 & 2.9384 & 4.7938 & 5.2449 & 0.7874 & -0.3466 & 0.4217 \\
& {$[0.000]$} & {$[0.000]$} & {$[0.001]$} & {$[0.002]$} & {$[0.709]$} & {$[0.823]$} & {$[0.010]$} \\
\hline \multirow{2}{*}{ Inflación } & -0.6290 & 0.0736 & 7.7512 & 6.9786 & 1.6089 & 1.7595 & 0.6337 \\
& {$[0.036]$} & {$[0.822]$} & {$[0.011]$} & {$[0.027]$} & {$[0.000]$} & {$[0.000]$} & {$[0.824]$} \\
\hline \multirow{2}{*}{ M1 } & 1.8656 & 1.6665 & 0.1865 & 0.2150 & -0.8896 & -1.4593 & 1.8584 \\
& {$[0.000]$} & {$[0.000]$} & {$[0.084]$} & {$[0.065]$} & {$[0.125]$} & {$[0.019]$} & {$[0.004]$} \\
\hline \multirow{2}{*}{ Producto } & -0.9154 & -0.9557 & -0.0527 & 0.0661 & -3.1398 & -2.5630 & 1.2973 \\
& {$[0.006]$} & {$[0.015]$} & {$[0.638]$} & {$[0.712]$} & {$[0.002]$} & {$[0.002]$} & {$[0.094]$} \\
\hline \multirow{2}{*}{ TIUSA } & -0.0278 & -5.3857 & -0.0445 & -0.0646 & -0.0647 & -0.2083 & -16.8228 \\
& {$[0.168]$} & {$[0.010]$} & {$[0.031]$} & {$[0.030]$} & {$[0.034]$} & {$[0.016]$} & {$[0.004]$} \\
\hline \multirow{2}{*}{ LIBOR } & - & 6.9774 & - & 0.0376 & - & 0.1718 & - \\
\hline \multirow{2}{*}{ Coeficiente } & -0.4519 & -0.3530 & -0.3041 & -0.2923 & -0.2124 & -0.2798 & -0.1462 \\
ajuste & {$[0.000]$} & {$[0.000]$} & {$[0.000]$} & {$[0.000]$} & {$[0.009]$} & {$[0.001]$} & {$[0.001]$} \\
\hline
\end{tabular}

Fuente: Elaboración de los autores con datos del International Financial Statistics y Banco de México. Estimaciones mediante un Modelo Autorregresivo con Rezagos Distribuidos (ARDL).

1/ Las tasas de interés foráneas para Reino Unidos son las prevalecientes en Estados Unidos y la Zona Euro; 2/ Para aproximar la tasa de interés extranjera empleamos el indicador LIBOR y el de la eurozona. Valor-p entre corchetes 
Por otro lado, a pesar de que los resultados para Alemania, Japón y Brasil no comprueban una depreciación súbita del tipo de cambio ante un choque monetario, las estimaciones muestran la presencia de un desajuste cambiario (apreciación) significativo de su senda de largo plazo, especialmente en el caso de la economía brasileña. Algunos de los factores condicionantes están relacionados el carácter semifijo de la paridad cambiaria en estos países, la reconversión (reprimarización) de la estructura comercial (Brasil), el estancamiento económico y la deflación (Japón).

Cuadro 4. Sobrerreacción del tipo de cambio y choques externos

\begin{tabular}{|c|c|c|c|c|c|c|c|}
\hline Variable & $U K^{1 /}$ & $D E U$ & $D E U$ & $J P N$ & $J P N$ & $U^{2} A^{2 /}$ & $U^{2 /}$ \\
\hline \multirow{2}{*}{ Constante } & 1.2204 & -29.8851 & -16.7592 & -3.7529 & -52.1115 & 24.7866 & -17.1268 \\
& {$[0.880]$} & {$[0.049]$} & {$[0.171]$} & {$[0.855]$} & {$[0.002]$} & {$[0.239]$} & {$[0.059]$} \\
\hline \multirow{2}{*}{ TID } & 0.2644 & -11.6115 & -10.6468 & -85.5639 & -122.2325 & 1.1930 & -2.0015 \\
& {$[0.005]$} & {$[0.004]$} & {$[0.009]$} & {$[0.013]$} & {$[0.000]$} & {$[0.482]$} & {$[0.194]$} \\
\hline \multirow{2}{*}{ Inflación } & 7.6447 & -4.2011 & -3.8641 & 7.7412 & 11.4509 & 2.0177 & -1.0922 \\
& {$[0.004]$} & {$[0.059]$} & {$[0.005]$} & {$[0.036]$} & {$[0.000]$} & {$[0.336]$} & {$[0.083]$} \\
\hline \multirow{2}{*}{ M1 } & 1.0555 & -0.5564 & -0.6205 & -0.3931 & -0.7692 & 1.0735 & 0.3586 \\
& {$[0.007]$} & {$[0.170]$} & {$[0.021]$} & {$[0.121]$} & {$[0.000]$} & {$[0.001]$} & {$[0.017]$} \\
\hline \multirow{2}{*}{ Producto } & 0.1036 & 3.7536 & 2.7962 & 0.6522 & 3.8996 & -1.9616 & 1.5298 \\
& {$[0.843]$} & {$[0.007]$} & {$[0.004]$} & {$[0.640]$} & {$[0.001]$} & {$[0.296]$} & {$[0.025]$} \\
\hline \multirow{2}{*}{ TIUSA } & -11.4569 & -0.0478 & 5.7926 & 2.9543 & -4.6211 & 0.1361 & 0.0748 \\
& {$[0.001]$} & {$[0.220]$} & {$[0.004]$} & {$[0.200]$} & {$[0.084]$} & {$[0.086]$} & {$[0.040]$} \\
\hline \multirow{2}{*}{ LIBOR } & -9.9898 & - & -8.0134 & - & 5.8215 & - & -0.0123 \\
& {$[0.001]$} & & {$[0.000]$} & & {$[0.028]$} & & {$[0.705]$} \\
\hline \multirow{2}{*}{ Coeficiente de } & -0.2161 & -0.1939 & -0.3141 & -0.1496 & -0.2407 & -0.1539 & -0.2444 \\
ajuste & {$[0.000]$} & {$[0.000]$} & {$[0.000]$} & {$[0.000]$} & {$[0.000]$} & {$[0.002]$} & {$[0.000]$} \\
\hline
\end{tabular}

Fuente: Elaboración de los autores con datos del Banco Mundial, OCDE, FMI y el Banco de la Reserva Federal. Nota 1. Estimaciones realizadas mediante un Modelo Autorregresivo con Rezagos Distribuidos (ARDL). 1/ Las tasas de interés foráneas para Reino Unido son las prevalecientes en Estados Unidos y la Zona Euro; 2/ Para aproximar la tasa de interés extranjera se emplea el indicador LIBOR y el de la eurozona. Valor-p entre corchetes

Efectivamente, los precios domésticos responden de manera significativa a los cambios en la oferta monetaria (véase gráfica 1), lo que confirma su carácter de mecanismo de ajuste de las desviaciones del tipo de cambio a su valor de equilibrio. Es decir, en la medida que los precios son menores a su valor de equilibrio, esto implicará que la convergencia a su senda de largo plazo induzca una contracción de la oferta real de dinero y, por ende, el aumento de la tasa de interés domestica $\left(i>i^{*}\right)$, con lo cual el tipo de cambio tenderá a apreciarse en torno de la condición de la paridad de interés descubierta. 
REMEF (The Mexican Journal of Economics and Finance)

La pandemia Covid-19, la crisis financiera y la dinámica (Overshooting) del tipo de cambio

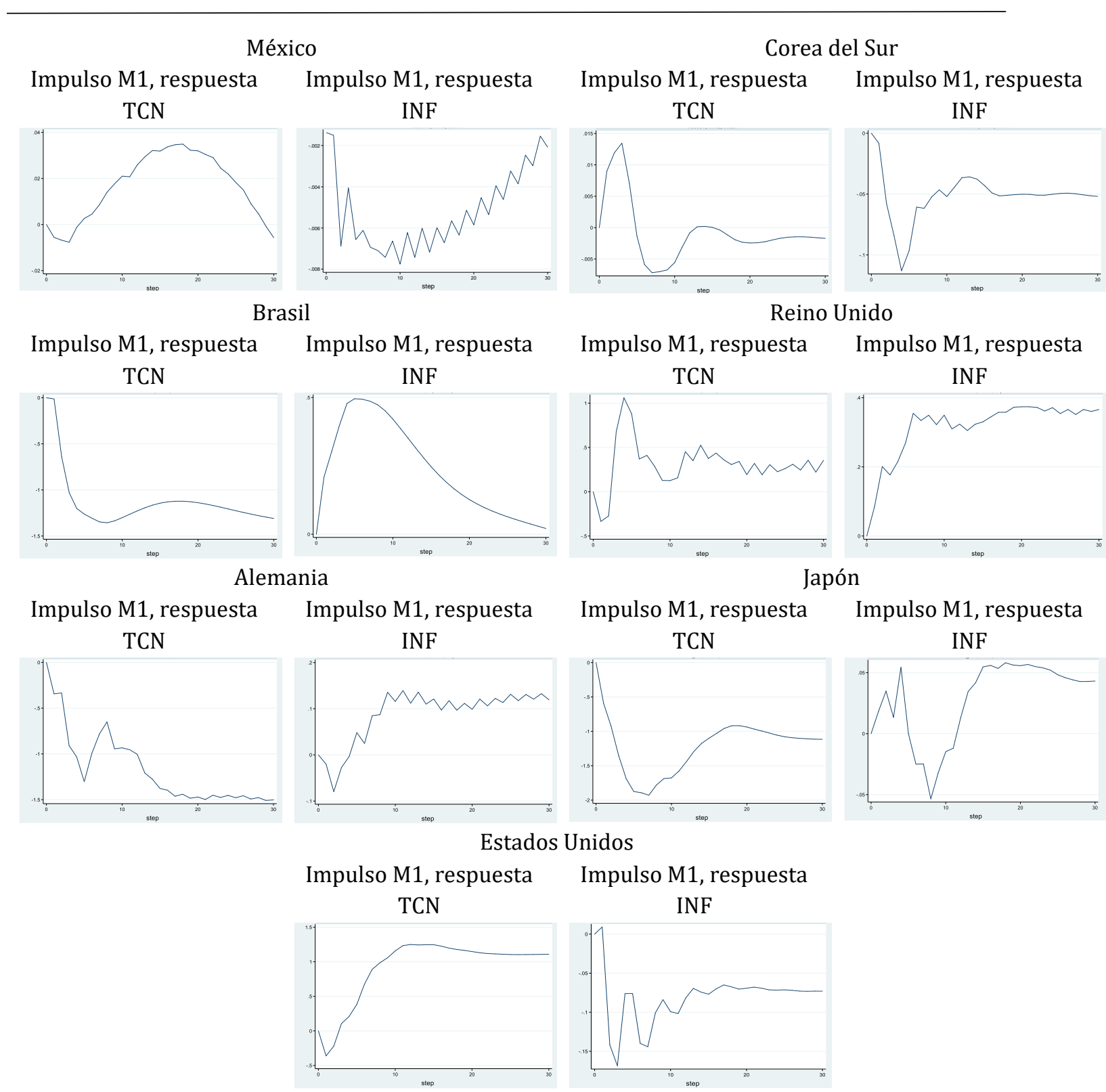

Grafica 1. Reacción del tipo de cambio y de la inflación ante desequilibrios monetarios.

Fuente: Elaboración de los autores con datos del Banco Mundial, OCDE, FMI y el Banco de la Reserva Federal. Nota: las estimaciones se realizaron con base en el método de Vectores Autorregresivos con Corrección de Error (VEC). Valor-p entre corchetes

Estos resultados confirman que, ante un choque en la oferta monetaria, el tipo de cambio nominal presenta una sobrerreacción en el corto plazo, superior a la depreciación requerida en el largo plazo, condición que provocará un incremento de precios y, con ello, un aumento gradual de la tasa de interés, entorno que inducirá un ingreso de capitales y una lenta apreciación cambiaria. 


\section{Conclusión}

En este artículo hemos evaluado la dinámica del tipo de cambio de Alemania, Brasil, Corea del Sur, Estados Unidos, Japón, México y Reino Unido durante el periodo 2000:01-2020:03. Nuestro objetivo fundamental fue comprobar la hipótesis de la existencia del fenómeno de overshooting como resultado de las intervenciones fiscales y monetarias expansivas adoptadas por los gobiernos y bancos centrales de esos países para confrontar los efectos recesivos de la crisis financiera de 20072008 y los choques de oferta y demanda derivados de la crisis sanitaria Covid-19.

La relación de largo plazo entre las variables relevantes fue analizada mediante un modelo ARDL; utilizamos el procedimiento de Johansen-Joselius para obtener funciones de impulsorespuesta y simular los efectos de una perturbación de corto plazo sobre el comportamiento del tipo de cambio y de los precios.

Empíricamente, nos interesó comprobar si el tipo de cambio nominal ha experimentado alteraciones significativas (sobrerreacción) respecto de su nivel de largo plazo ante un cambio no anticipado de la oferta monetaria, donde la variación de los precios internos es el mecanismo de ajuste que permite a la paridad cambiaria retornar a su senda de equilibrio (Dornbusch, 1976).

En general, las estimaciones confirman que las variaciones de la oferta monetaria provocan choques temporales (depreciación) sobre el tipo de cambio en Corea del Sur, México, Reino Unido y Estados Unidos. El deslizamiento cambiario es más profundo en el caso del peso mexicano, la libra esterlina y el dólar estadounidense, entre otros motivos, por la disímil velocidad de ajuste entre el mercado de bienes y el de activos, el volumen de negociación de estas divisas en los mercados internacionales, la especulación financiera, las presiones fiscales y las de la deuda.

Aunque los resultados para Alemania, Japón y Brasil no comprueban la existencia de una depreciación súbita del tipo de cambio ante un choque monetario, las estimaciones muestran la presencia de un desajuste cambiario (apreciación) significativo con respecto de la senda de largo plazo, especialmente en el caso de Brasil. Algunos de los factores condicionantes en estos casos están relacionados ya sea con el carácter semifijo de la paridad cambiaria (Alemania), o con la reconversión o reprimarización de la estructura comercial (Brasil) o con el estancamiento económico y la deflación (Japón).

Estos resultados confirman que, ante un choque en la oferta monetaria, el tipo de cambio nominal presenta una sobrerreacción en el corto plazo, superior a la depreciación requerida en el largo plazo, condición que provocará un incremento de precios y, con ello, un aumento gradual de la tasa de interés, entorno que inducirá un ingreso de capitales y una lenta apreciación cambiaria. Dada la centralidad de la variable tipo de cambio en una economía abierta, los efectos en la estabilidad cambiaria de las políticas macroeconómicas expansivas para paliar las crisis -tanto la que procede de la turbulencia financiera como la inducida por la pandemia- repercuten en el perfil de deuda externa, en la hoja de balance de los hogares, de las corporaciones financieras y no financieras y en la balanza de pagos y el crecimiento económico de largo plazo. Finalmente, a partir de este análisis pueden derivarse ulteriores investigaciones que exceden el objetivo de este trabajo, por ejemplo, el escrutinio de las consecuencias del overshooting del tipo de cambio en economías con pasivos en moneda extranjera, en especial en el caso de economías emergentes que, por la naturaleza de la estructura de la deuda, la inestabilidad cambiaria (depreciación) puede crear condiciones significativas de fragilidad financiera. Otra línea de investigación posible, se relaciona con el efecto 
del overshooting (devaluación monetaria) en la cuenta corriente de la balanza de pagos en virtud del cumplimiento de la condición Marshall-Lerner que consiste en la elasticidad positiva de las exportaciones y las importaciones ante variaciones del tipo de cambio.

\section{Referencias}

[1] Aguilar, A. y C. Cantú (2020), Monetary policy response in emerging market economies: why was it different this time? Bank for International Settlements Bulletin, 32, 1-7.

[2] Baldwin, R. y Weder di Mauro, B.(eds.). (2020). Mitigating the COVID Economic Crisis: Act fast and do whatever it takes. Eds. Center of Economic Policy Research CEPR Press.

[3] Bhandari, J. S. (1982). Exchange Rate Determination and Adjustment. Nueva York: Praeger Publishers, Inc.

[4] BIS (Bank for International Settlements) (2020), BIS Quarterly Review, Basel, September [online] https://www.bis.org/publ/qtrpdf/r_qt2009.htm.

[5] _ (2020b), "Global liquidity indicators", 26 October [online] https://www.bis.org/statistics/gli.htm.

[6] _ (2020c), "Debt securities statistics", $14 \quad$ September [online] https://www.bis.org/statistics/secstats.htm.

[7] Blackburne, E., and Frank, M. (2007). Estimation of nonstationary heterogeneous panels. The Stata Journal, 7(2), 197-208. https://doi.org/10.1177/1536867X0700700204

[8] Board of Governors of the Federal Reserve System (2020). Federal Reserve Board announces the extensions of its temporary U.S. dollar liquidity swap lines and the temporary repurchase agreement facility for foreign and international monetary authorities (FIMA repo facility) through March 31, 2021. 29 July [online] https://www.federalreserve.gov/newsevents/pressreleases/monetary20200729b.htm.

[9] Capistrán, C., Chiquiar, D. and Hernández, J. (2019). Identifying Dornbusch's exchange rate overshooting with structural VECs: evidence from Mexico. International Journal of Central Banking, 15 (5), 207-254. https://doi.org/10.36095/banxico/di.2017.11

[10] Congressional Budget Office. (2021). CBO's Budget and Economic Outlook. Washington D.C.

[11] Dornbusch, R. (1976). Expectations and Exchange Rate Dynamics. Journal of Political Economy, 84(6), 1161-1176. https://doi.org/10.1086/260506

[12] FMI. (2021). World Economic Outlook, enero.

[13] Dornbusch, R., Park, Y. y Claessens, S. (2000). Contagion: Understanding how it spreads. The World Bank Research Observer, 15, 197-165. https://doi.org/10.1093/wbro/15.2.177

[14] Ghosh, T. y Bhadury, S. (2017). Exchange Rate Overshooting: A Reassessment in a Monetary Framework. The Empirical Economics Letters, 16 (11), 1143-1149.

[15] Güneş, S. y Karul, Ç. (2016). The Exchange Rate Overshooting in Turkey, Pamurkale Journal of Euroasian Economics Studies, 3(1), 27-35. https://doi.org/10.5505/pjess.2016.02986

[16] Hetzel, R. L. (2008). The Monetary Policy of the Federal Reserve. Cambridge, R.U.: Cambridge University Press. https://doi.org/10.1017/СB09780511754173

[17] Kim,J., Kim, S. y Park, D. (2020). Monetary policy shocks and exchange rates in Asian countries. Japan and the World Economy, 56, 1-19. https://doi.org/10.1016/j.japwor.2020.101041

[18] Locker, P. (1980). "Statement", en Recent Monetary Policy Developments. Washington D.C.: House Committee on Banking, Finance, and Urban Affairs, Subcommittee on Domestic Monetary Policy, 96th Congress, $2^{\text {a }}$ sesión, noviembre 19. 
[19] Niki, R. (2020). Medical and Social Security Reform after the Corona Crisis. Keiso Shobo.

[20] Park, S. (2020). The COVID-19 Economic Crisis and Policy Responses. Monthly CCEJ, May-June. Available at: ccej.or.kr/61674

[21] Perrotini, I. (2015). La reserva federal, la crisis y la política monetaria no convencional, Contaduría y Administración, 60 (S2), 250-271. https://doi.org/10.1016/j.cya.2015.11.001

[22] Pesaran, H., Shin, Y. y Smith, R. (1999). Pooled Mean Group Estimation of Dynamic Heterogeneous Panels. Journal of the American Statistical Association, 94(446), 621-634. https://doi.org/10.1080/01621459.1999.10474156

[23] Pesaran, M. H., Shin, Y. \& Smith, R. J. (2001). Bounds testing approaches to the analysis of level relationships. Journal of applied econometrics, 16 (3), 289-326. https://doi.org/10.1002/jae.616

[24] Rüth, S. (2020). Shifts in monetary policy and exchange rate dynamics: Is Dornbusch's overshooting hypothesis intact, after all? Journal of International Economics, 126, 1-18. https://doi.org/10.1016/j.jinteco.2020.103344

[25] Sharifi-Renani, H., Raki M. y Honarvar, N. (2014). Monetary Policy and Exchange Rate Overshooting in Iran, International Economic Studies, 44 (1), 67-74. https://doi.org/10.22108/IES.2014.15572

[26] Thirlwall, A. P. (2020). The Age of Fragmentation by Alessandro Roncaglia: A Review Article. PSL Quarterly Review, 73 (295), 343-365. https://doi.org/10.13133/2037-3643_73.295_4

[27] Volcker, P. (1980). "Statement" en Recent Monetary Policy Developments. House Committee on Banking, Finance, and Urban Affairs, 96 ${ }^{\text {th }}$ Congress, $2^{\text {nd }}$ session, November 19.

\section{Anexo}

Pruebas de consistencia correspondientes a las regresiones ARDL

\section{Cuadro A.1. Orden de integración}

\section{Prueba de raíz unitaria}

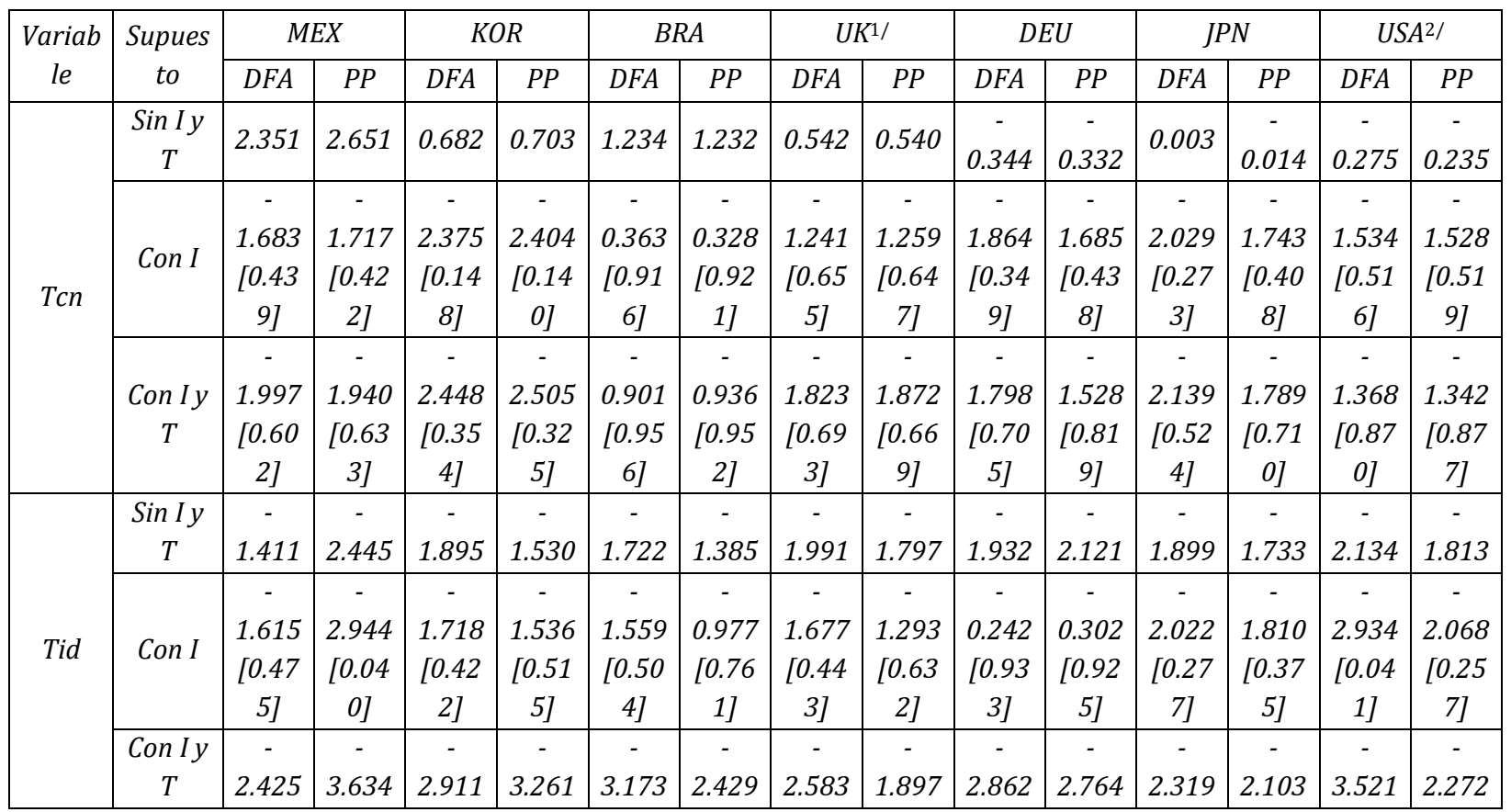


REMEF (The Mexican Journal of Economics and Finance)

La pandemia Covid-19, la crisis financiera y la dinámica (Overshooting) del tipo de cambio

\begin{tabular}{|c|c|c|c|c|c|c|c|c|c|c|c|c|c|c|c|}
\hline & & $\begin{array}{l}0.36 \\
6]\end{array}$ & $\begin{array}{c}{[0.02} \\
7]\end{array}$ & $\begin{array}{c}{[0.15} \\
8]\end{array}$ & \begin{tabular}{|c|}
{$[0.07$} \\
$2]$
\end{tabular} & $\begin{array}{c}{[0.08} \\
9]\end{array}$ & \begin{tabular}{|c}
{$[0.36$} \\
$4]$
\end{tabular} & $\begin{array}{c}{[0.28} \\
7]\end{array}$ & $\begin{array}{c}{[0.65} \\
6]\end{array}$ & $\begin{array}{c}{[0.17} \\
5]\end{array}$ & $\begin{array}{c}{[0.21} \\
0]\end{array}$ & \begin{tabular}{|c|}
{$[0.42$} \\
$3]$
\end{tabular} & $\begin{array}{c}{[0.54} \\
4]\end{array}$ & $\begin{array}{c}{[0.03} \\
7]\end{array}$ & $\begin{array}{c}{[0.44} \\
9]\end{array}$ \\
\hline \multirow{3}{*}{$I p c$} & $\begin{array}{c}\operatorname{Sin} I y \\
T\end{array}$ & 428 & 912 & 322 & 6.561 & 2.382 & 8.999 & 137 & 1.094 & 2.675 & 8.124 & 0.481 & 0.463 & 4.505 & 7.893 \\
\hline & Con I & $\begin{array}{c}2.898 \\
{[0.04} \\
5]\end{array}$ & $\begin{array}{c}5.075 \\
{[0.00} \\
0]\end{array}$ & $\begin{array}{c}3.927 \\
{[0.00} \\
1]\end{array}$ & \begin{tabular}{|c}
7.392 \\
{$[0.00$} \\
$0]$
\end{tabular} & $\begin{array}{c}- \\
1.597 \\
{[0.48} \\
5] \\
\end{array}$ & \begin{tabular}{|c}
2.183 \\
{$[0.21$} \\
$2]$ \\
\end{tabular} & $\begin{array}{c}- \\
2.867 \\
{[0.04} \\
9] \\
\end{array}$ & $\begin{array}{c}2.412 \\
{[0.13} \\
8]\end{array}$ & \begin{tabular}{|c}
1.566 \\
{$[0.50$} \\
$0]$ \\
\end{tabular} & $\begin{array}{c}1.495 \\
{[0.53} \\
5]\end{array}$ & $\begin{array}{c}- \\
0.925 \\
{[0.77} \\
9]\end{array}$ & $\begin{array}{c}- \\
0.718 \\
{[0.84} \\
2]\end{array}$ & $\begin{array}{c}2.033 \\
{[0.27} \\
2] \\
\end{array}$ & \begin{tabular}{|c}
- \\
2.071 \\
{$[0.25$} \\
$6]$
\end{tabular} \\
\hline & $\begin{array}{c}\text { Con Iy } \\
T\end{array}$ & $\begin{array}{c}- \\
1.987 \\
{[0.60} \\
8]\end{array}$ & $\begin{array}{c}- \\
2.113 \\
{[0.53} \\
8]\end{array}$ & $\begin{array}{c}1.177 \\
{[0.91} \\
5]\end{array}$ & \begin{tabular}{|c|}
- \\
2.388 \\
{$[0.38$} \\
$6]$
\end{tabular} & $\begin{array}{c}- \\
1.830 \\
{[0.69} \\
0]\end{array}$ & \begin{tabular}{|c|}
- \\
1.447 \\
{$[0.84$} \\
$6]$
\end{tabular} & $\begin{array}{c}- \\
2.810 \\
{[0.19} \\
3]\end{array}$ & $\begin{array}{c}2.358 \\
{[0.40} \\
2]\end{array}$ & \begin{tabular}{|c|}
- \\
1.703 \\
{$[0.74$} \\
$9]$
\end{tabular} & $\begin{array}{c}1.959 \\
{[0.62} \\
3]\end{array}$ & \begin{tabular}{|c}
- \\
1.834 \\
{$[0.68$} \\
$8]$
\end{tabular} & $\begin{array}{c}- \\
1.999 \\
{[0.60} \\
1]\end{array}$ & $\begin{array}{c}- \\
1.558 \\
{[0.80} \\
8]\end{array}$ & $\begin{array}{c}- \\
1.736 \\
{[0.73} \\
4]\end{array}$ \\
\hline \multirow{3}{*}{$m 1$} & $\begin{array}{c}\text { Sin I } y \\
T\end{array}$ & 1.131 & 1.640 & 2.329 & 2.660 & 0.901 & 1.074 & 3.643 & 3.541 & 3.242 & 5.140 & 2.241 & 3.693 & 0.861 & 0.987 \\
\hline & Con I & $\begin{array}{c}- \\
1.741 \\
{[0.41} \\
0]\end{array}$ & $\begin{array}{c}- \\
2.127 \\
{[0.23} \\
3]\end{array}$ & $\begin{array}{c}0.091 \\
{[0.96} \\
5]\end{array}$ & \begin{tabular}{|c}
0.417 \\
{$[0.98$} \\
$2]$
\end{tabular} & $\begin{array}{c}- \\
2.210 \\
{[0.20} \\
2]\end{array}$ & \begin{tabular}{|c}
- \\
1.914 \\
{$[0.32$} \\
$5]$
\end{tabular} & $\begin{array}{c}- \\
0.733 \\
{[0.83} \\
8]\end{array}$ & \begin{tabular}{|c}
- \\
0.897 \\
{$[0.78$} \\
$9]$
\end{tabular} & \begin{tabular}{|c}
- \\
0.488 \\
{$[0.89$} \\
$4]$
\end{tabular} & $\begin{array}{c}- \\
0.301 \\
{[0.92} \\
5]\end{array}$ & \begin{tabular}{|c|}
- \\
1.118 \\
{$[0.70$} \\
$7]$
\end{tabular} & $\begin{array}{c}- \\
1.462 \\
{[0.55} \\
2]\end{array}$ & $\begin{array}{c}0.159 \\
{[0.96} \\
9]\end{array}$ & $\begin{array}{c}0.523 \\
{[0.98} \\
5]\end{array}$ \\
\hline & $\begin{array}{c}\text { on Iy } \\
T\end{array}$ & $\begin{array}{c}- \\
4.314 \\
{[0.00} \\
3] \\
\end{array}$ & $\begin{array}{c}- \\
3.688 \\
{[0.02} \\
3]\end{array}$ & $\begin{array}{c}- \\
1.433 \\
{[0.85} \\
0]\end{array}$ & \begin{tabular}{|c|}
- \\
1.208 \\
{$[0.90$} \\
$8]$ \\
\end{tabular} & \begin{tabular}{|c}
- \\
2.215 \\
{$[0.48$} \\
$1]$ \\
\end{tabular} & \begin{tabular}{|c|}
- \\
1.920 \\
{$[0.64$} \\
$4]$ \\
\end{tabular} & $\begin{array}{c}- \\
2.449 \\
{[0.35} \\
3] \\
\end{array}$ & \begin{tabular}{|c|}
- \\
2.784 \\
{$[0.20$} \\
$3]$ \\
\end{tabular} & \begin{tabular}{|c|}
- \\
1.952 \\
{$[0.62$} \\
$7]$ \\
\end{tabular} & $\begin{array}{c}- \\
2.079 \\
{[0.55} \\
7] \\
\end{array}$ & $\begin{array}{c}- \\
2.533 \\
{[0.31} \\
1] \\
\end{array}$ & $\begin{array}{c}- \\
2.429 \\
{[0.36} \\
4]\end{array}$ & $\begin{array}{c}- \\
1.164 \\
{[0.91} \\
7] \\
\end{array}$ & $\begin{array}{c}- \\
1.026 \\
{[0.94} \\
0]\end{array}$ \\
\hline \multirow{3}{*}{$P i b$} & $\begin{array}{c}\operatorname{Sin} I y \\
T\end{array}$ & 2.151 & 2.667 & 4.563 & \begin{tabular}{|l|l}
7.885 \\
\end{tabular} & 2.523 & 2.569 & 0.236 & $\mid 1.139$ & 1.594 & $\mid 1.511$ & 1.016 & 0.904 & 2.501 & 3.427 \\
\hline & Con I & $\begin{array}{c}1.752 \\
{[0.40} \\
4]\end{array}$ & $\begin{array}{c}1.823 \\
{[0.36} \\
9]\end{array}$ & $\begin{array}{c}3.104 \\
{[0.02} \\
6]\end{array}$ & \begin{tabular}{|c}
4.064 \\
{$[0.00$} \\
$1]$
\end{tabular} & $\begin{array}{c}2.209 \\
{[0.20} \\
3]\end{array}$ & \begin{tabular}{|c}
2.155 \\
{$[0.22$} \\
$3]$ \\
\end{tabular} & $\begin{array}{c}1.958 \\
{[0.30} \\
5]\end{array}$ & $\begin{array}{c}2.058 \\
{[0.26} \\
1]\end{array}$ & $\begin{array}{c}1.283 \\
{[0.63} \\
6]\end{array}$ & $\begin{array}{c}1.219 \\
{[0.66} \\
5]\end{array}$ & \begin{tabular}{|c}
1.977 \\
{$[0.29$} \\
$6]$
\end{tabular} & $\begin{array}{c}2.141 \\
{[0.22} \\
8]\end{array}$ & $\begin{array}{c}- \\
1.465 \\
{[0.55} \\
0]\end{array}$ & $\begin{array}{c}- \\
1.144 \\
{[0.69} \\
7]\end{array}$ \\
\hline & $\begin{array}{c}\text { Con Iy } \\
T\end{array}$ & $\begin{array}{c}1.965 \\
{[0.62} \\
0]\end{array}$ & $\begin{array}{c}- \\
2.738 \\
{[0.22} \\
0]\end{array}$ & $\begin{array}{c}1.626 \\
{[0.78} \\
2]\end{array}$ & \begin{tabular}{|c}
1.355 \\
{$[0.87$} \\
$3]$
\end{tabular} & $\begin{array}{c}0.013 \\
{[0.99} \\
4]\end{array}$ & \begin{tabular}{|c}
0.383 \\
{$[0.98$} \\
$7]$
\end{tabular} & $\begin{array}{c}0.887 \\
{[0.95} \\
7]\end{array}$ & $\begin{array}{c}3.384 \\
{[0.05} \\
3]\end{array}$ & $\begin{array}{c}2.581 \\
{[0.28} \\
8]\end{array}$ & $\begin{array}{c}3.231 \\
{[0.07} \\
8]\end{array}$ & $\begin{array}{c}2.259 \\
{[0.45} \\
7]\end{array}$ & $\begin{array}{c}2.909 \\
{[0.15} \\
9]\end{array}$ & $\begin{array}{c}1.974 \\
{[0.61} \\
5]\end{array}$ & $\begin{array}{c}2.767 \\
{[0.20} \\
9]\end{array}$ \\
\hline \multirow{3}{*}{ tiusa } & $\begin{array}{c}\text { Sin } I y \\
T\end{array}$ & .134 & 813 & 134 & 1.813 & 2.134 & 1.813 & $\begin{array}{c}- \\
2.134\end{array}$ & 813 & 2.134 & 1.813 & 2.134 & $\begin{array}{c}- \\
1.813\end{array}$ & 827 & $\begin{array}{c}- \\
1.891\end{array}$ \\
\hline & Con I & $\begin{array}{c}2.934 \\
{[0.04} \\
1]\end{array}$ & $\begin{array}{c}- \\
2.068 \\
{[0.25} \\
7]\end{array}$ & $\begin{array}{c}2.934 \\
{[0.04} \\
1] \\
\end{array}$ & \begin{tabular}{|c}
- \\
2.068 \\
{$[0.25$} \\
$7]$ \\
\end{tabular} & $\begin{array}{c}- \\
2.934 \\
{[0.04} \\
1] \\
\end{array}$ & \begin{tabular}{|c}
2.068 \\
{$[0.25$} \\
$7]$ \\
\end{tabular} & $\begin{array}{c}- \\
2.934 \\
{[0.04} \\
1] \\
\end{array}$ & $\begin{array}{c}2.068 \\
{[0.25} \\
7] \\
\end{array}$ & \begin{tabular}{|c}
- \\
2.934 \\
{$[0.04$} \\
$1]$ \\
\end{tabular} & \begin{tabular}{|c}
- \\
2.068 \\
{$[0.25$} \\
$7]$ \\
\end{tabular} & $\begin{array}{c}2.934 \\
{[0.04} \\
1] \\
\end{array}$ & $\begin{array}{c}- \\
2.068 \\
{[0.25} \\
7]\end{array}$ & $\begin{array}{c}0.367 \\
{[0.98} \\
0]\end{array}$ & $\begin{array}{c}- \\
0.165 \\
{[0.94} \\
2]\end{array}$ \\
\hline & $\begin{array}{c}\text { Con Iy } \\
T\end{array}$ & $\begin{array}{c}- \\
3.521 \\
{[0.03} \\
7]\end{array}$ & $\begin{array}{c}- \\
2.272 \\
{[0.44} \\
9]\end{array}$ & $\begin{array}{c}3.521 \\
{[0.03} \\
7]\end{array}$ & $\begin{array}{c}2.272 \\
{[0.44} \\
9]\end{array}$ & $\begin{array}{c}- \\
3.521 \\
{[0.03} \\
7]\end{array}$ & \begin{tabular}{|c}
- \\
2.272 \\
{$[0.44$} \\
$9]$
\end{tabular} & $\begin{array}{c}- \\
3.521 \\
{[0.03} \\
7]\end{array}$ & \begin{tabular}{|c}
- \\
2.272 \\
{$[0.44$} \\
$9]$
\end{tabular} & \begin{tabular}{|c}
- \\
3.521 \\
{$[0.03$} \\
$7]$
\end{tabular} & $\begin{array}{c}- \\
2.272 \\
{[0.44} \\
9]\end{array}$ & \begin{tabular}{|c}
- \\
3.521 \\
{$[0.03$} \\
$7]$
\end{tabular} & $\begin{array}{c}2.272 \\
{[0.44} \\
9]\end{array}$ & $\begin{array}{c}1.646 \\
{[0.77} \\
3]\end{array}$ & $\begin{array}{c}- \\
2.066 \\
{[0.56} \\
4]\end{array}$ \\
\hline \multirow{3}{*}{ libor } & $\begin{array}{c}\operatorname{Sin} I y \\
T\end{array}$ & 2.028 & $\begin{array}{c}- \\
1.851\end{array}$ & $\begin{array}{c}- \\
2.028\end{array}$ & $\begin{array}{c}- \\
1.851\end{array}$ & $\begin{array}{c}- \\
2.028\end{array}$ & \begin{tabular}{|c|}
- \\
1.851
\end{tabular} & $\begin{array}{c}- \\
1.827\end{array}$ & $\mid$\begin{tabular}{|l|l}
1.891 \\
\end{tabular} & $\begin{array}{c}- \\
2.028\end{array}$ & $\begin{array}{c}- \\
1.851\end{array}$ & \begin{tabular}{|c|}
- \\
2.028
\end{tabular} & $\begin{array}{c}- \\
1.851\end{array}$ & $\begin{array}{c}- \\
2.028\end{array}$ & \begin{tabular}{|c|}
- \\
1.851
\end{tabular} \\
\hline & Con I & $\begin{array}{c}- \\
1.802 \\
{[0.37} \\
9]\end{array}$ & $\begin{array}{c}- \\
1.220 \\
{[0.66} \\
5]\end{array}$ & $\begin{array}{c}- \\
1.802 \\
{[0.37} \\
9]\end{array}$ & $\begin{array}{c}- \\
1.220 \\
{[0.66} \\
5]\end{array}$ & $\begin{array}{c}- \\
1.802 \\
{[0.37} \\
9]\end{array}$ & $\begin{array}{c}- \\
1.220 \\
{[0.66} \\
5]\end{array}$ & $\begin{array}{c}0.367 \\
{[0.98} \\
0]\end{array}$ & $\begin{array}{c}- \\
0.165 \\
{[0.94} \\
2]\end{array}$ & $\begin{array}{c}- \\
1.802 \\
{[0.37} \\
9]\end{array}$ & $\begin{array}{c}- \\
1.220 \\
{[0.66} \\
5]\end{array}$ & \begin{tabular}{|c}
- \\
1.802 \\
{$[0.37$} \\
$9]$
\end{tabular} & $\begin{array}{c}- \\
1.220 \\
{[0.66} \\
5]\end{array}$ & $\begin{array}{c}- \\
1.802 \\
{[0.37} \\
9]\end{array}$ & $\begin{array}{c}- \\
1.220 \\
{[0.66} \\
5]\end{array}$ \\
\hline & $\begin{array}{c}\text { Con Iy } \\
T\end{array}$ & $\begin{array}{c}- \\
2.773 \\
\end{array}$ & .706 & $\begin{array}{l}- \\
773\end{array}$ & \begin{tabular}{|c|} 
\\
1.706
\end{tabular} & $\begin{array}{c}- \\
2.773\end{array}$ & \begin{tabular}{|c|} 
\\
1.706
\end{tabular} & $\begin{array}{c}- \\
1.646\end{array}$ & $\begin{array}{c}- \\
2.066\end{array}$ & $\begin{array}{c}- \\
2.773\end{array}$ & \begin{tabular}{|c|}
- \\
1.706
\end{tabular} & $\begin{array}{c}- \\
2.773 \\
\end{array}$ & $\begin{array}{c}- \\
1.706\end{array}$ & $\begin{array}{c}- \\
2.773\end{array}$ & $\begin{array}{c}- \\
1.706\end{array}$ \\
\hline
\end{tabular}




\begin{tabular}{|c|c|c|c|c|c|c|c|c|c|c|c|c|c|c|c|}
\hline & & $\begin{array}{c}{[0.20} \\
6]\end{array}$ & \begin{tabular}{|c|}
0.74 \\
$8]$
\end{tabular} & $\begin{array}{c}{[0.20} \\
6]\end{array}$ & $\begin{array}{c}0.74 \\
8]\end{array}$ & $\begin{array}{c}{[0.20} \\
6]\end{array}$ & $\begin{array}{c}{[0.74} \\
8]\end{array}$ & $\begin{array}{c}{[0.77} \\
3]\end{array}$ & $\begin{array}{c}{[0.56} \\
4]\end{array}$ & $\begin{array}{c}{[0.20} \\
6]\end{array}$ & $\begin{array}{c}{[0.74} \\
8]\end{array}$ & $\begin{array}{c}{[0.20} \\
6]\end{array}$ & $\begin{array}{c}{[0.74} \\
8]\end{array}$ & $\begin{array}{c}{[0.20} \\
6]\end{array}$ & $\begin{array}{c}{[0.74} \\
8]\end{array}$ \\
\hline \multicolumn{16}{|c|}{ Primeras diferencias } \\
\hline \multirow{3}{*}{$d t c n$} & $\begin{array}{c}\text { in I y } \\
T\end{array}$ & 9.281 & $\begin{array}{c}- \\
9.281\end{array}$ & $\begin{array}{c}- \\
4.929\end{array}$ & 8.296 & - & 6.104 & $\begin{array}{c}- \\
4.811\end{array}$ & 6.144 & $\begin{array}{c}- \\
6.128\end{array}$ & $\begin{array}{c}- \\
6.355\end{array}$ & $\begin{array}{c}- \\
3.908\end{array}$ & $\begin{array}{c}- \\
7.201\end{array}$ & 5.036 & $\begin{array}{c}- \\
6.141\end{array}$ \\
\hline & Con I & $\begin{array}{c}9.848 \\
{[0.00} \\
0]\end{array}$ & $\begin{array}{c}9.848 \\
{[0.00} \\
0]\end{array}$ & $\begin{array}{c}4.980 \\
{[0.00} \\
0]\end{array}$ & $\begin{array}{c}8.290 \\
{[0.00} \\
0]\end{array}$ & $\begin{array}{c}5.447 \\
{[0.00} \\
0]\end{array}$ & $\begin{array}{c}6.172 \\
{[0.00} \\
0]\end{array}$ & $\begin{array}{c}4.813 \\
{[0.00} \\
0]\end{array}$ & $\begin{array}{c}6.125 \\
{[0.00} \\
0]\end{array}$ & $\begin{array}{c}6.099 \\
{[0.00} \\
0]\end{array}$ & $\begin{array}{c}6.324 \\
{[0.00} \\
0]\end{array}$ & $\begin{array}{c}3.877 \\
{[0.00} \\
2]\end{array}$ & $\begin{array}{c}7.153 \\
{[0.00} \\
0]\end{array}$ & $\begin{array}{c}5.011 \\
{[0.00} \\
0]\end{array}$ & $\begin{array}{c}6.107 \\
{[0.00} \\
0]\end{array}$ \\
\hline & $\begin{array}{c}\text { on Iy } \\
T\end{array}$ & $\begin{array}{c}9.889 \\
{[0.00} \\
0]\end{array}$ & $\begin{array}{c}9.889 \\
{[0.00} \\
0]\end{array}$ & $\begin{array}{c}5.019 \\
{[0.00} \\
0]\end{array}$ & $\begin{array}{c}8.278 \\
{[0.00} \\
0]\end{array}$ & $\begin{array}{c}5.522 \\
{[0.00} \\
0]\end{array}$ & $\begin{array}{c}6.217 \\
{[0.00} \\
0]\end{array}$ & $\begin{array}{c}- \\
4.807 \\
{[0.00} \\
0]\end{array}$ & $\begin{array}{c}6.091 \\
{[0.00} \\
0]\end{array}$ & $\begin{array}{c}6.095 \\
{[0.00} \\
0]\end{array}$ & $\begin{array}{c}6.304 \\
{[0.00} \\
0]\end{array}$ & $\begin{array}{c}3.816 \\
{[0.01} \\
5]\end{array}$ & $\begin{array}{c}- \\
7.093 \\
{[0.00} \\
0]\end{array}$ & $\begin{array}{c}5.028 \\
{[0.00} \\
0]\end{array}$ & $\begin{array}{c}- \\
6.077 \\
{[0.00} \\
0]\end{array}$ \\
\hline \multirow{3}{*}{ Dtid } & $\begin{array}{c}\operatorname{Sin} I y \\
T\end{array}$ & 5.072 & $\begin{array}{c}10.22 \\
7\end{array}$ & 7.127 & 8.234 & 4.718 & 4.613 & 4.682 & 4.951 & 5.147 & 7.096 & $\begin{array}{c}- \\
5.739\end{array}$ & $\begin{array}{c}- \\
6.039\end{array}$ & 3.911 & 6.974 \\
\hline & Con I & $\begin{array}{c}- \\
5.067 \\
{[0.00} \\
0]\end{array}$ & $\begin{array}{c}- \\
10.22 \\
8 \\
{[0.00} \\
0]\end{array}$ & $\begin{array}{c}- \\
7.204 \\
{[0.00} \\
0]\end{array}$ & $\begin{array}{c}- \\
8.250 \\
{[0.00} \\
0]\end{array}$ & $\begin{array}{c}- \\
4.901 \\
{[0.00} \\
0]\end{array}$ & $\begin{array}{c}- \\
4.668 \\
{[0.00} \\
0]\end{array}$ & $\begin{array}{c}- \\
4.823 \\
{[0.00} \\
0]\end{array}$ & $\begin{array}{c}- \\
5.068 \\
{[0.00} \\
0]\end{array}$ & $\begin{array}{c}- \\
5.691 \\
{[0.00} \\
0]\end{array}$ & $\begin{array}{c}- \\
7.479 \\
{[0.00} \\
0]\end{array}$ & $\begin{array}{c}- \\
5.708 \\
{[0.00} \\
0]\end{array}$ & $\begin{array}{c}- \\
6.007 \\
{[0.00} \\
0]\end{array}$ & $\begin{array}{c}- \\
3.947 \\
{[0.00} \\
1]\end{array}$ & $\begin{array}{c}- \\
6.982 \\
{[0.00} \\
0]\end{array}$ \\
\hline & $\begin{array}{c}\text { Con Iy } \\
T\end{array}$ & $\begin{array}{c}- \\
5.026 \\
{[0.00} \\
0]\end{array}$ & $\begin{array}{c}- \\
10.21 \\
5 \\
{[0.00} \\
0]\end{array}$ & $\begin{array}{c}- \\
7.190 \\
{[0.00} \\
0]\end{array}$ & $\begin{array}{c}- \\
8.218 \\
{[0.00} \\
0]\end{array}$ & $\begin{array}{c}- \\
4.861 \\
{[0.00} \\
0]\end{array}$ & $\begin{array}{c}- \\
4.674 \\
{[0.00} \\
0]\end{array}$ & $\begin{array}{c}- \\
4.809 \\
{[0.00} \\
0]\end{array}$ & $\begin{array}{c}- \\
5.048 \\
{[0.00} \\
0]\end{array}$ & $\begin{array}{c}- \\
5.652 \\
{[0.00} \\
0]\end{array}$ & $\begin{array}{c}- \\
7.429 \\
{[0.00} \\
0]\end{array}$ & $\begin{array}{c}- \\
5.716 \\
{[0.00} \\
0]\end{array}$ & $\begin{array}{c}- \\
6.004 \\
{[0.00} \\
0]\end{array}$ & $\begin{array}{c}- \\
3.960 \\
{[0.01} \\
0]\end{array}$ & $\begin{array}{c}- \\
6.942 \\
{[0.00} \\
0]\end{array}$ \\
\hline \multirow{3}{*}{$\operatorname{dipc}$} & $\begin{array}{c}\operatorname{Sin} I y \\
T\end{array}$ & $\begin{array}{c}- \\
2.103\end{array}$ & $\begin{array}{c}- \\
2.973\end{array}$ & $\begin{array}{c}- \\
2.454\end{array}$ & $\begin{array}{c}- \\
5.706\end{array}$ & $\begin{array}{c}- \\
1.448\end{array}$ & $\begin{array}{c}- \\
2.183\end{array}$ & $\begin{array}{c}- \\
5.689\end{array}$ & $\begin{array}{c} \\
6.405 \\
\end{array}$ & $\begin{array}{c}- \\
1.383\end{array}$ & \begin{tabular}{|c|} 
\\
6.189
\end{tabular} & $\begin{array}{c}- \\
3.437\end{array}$ & $\begin{array}{c}- \\
8.890\end{array}$ & $\begin{array}{c}- \\
2.009\end{array}$ & $\begin{array}{c}- \\
5.281\end{array}$ \\
\hline & Con I & $\begin{array}{c}- \\
2.834 \\
{[0.05} \\
3]\end{array}$ & $\begin{array}{c}- \\
4.292 \\
{[0.00} \\
0]\end{array}$ & $\begin{array}{c}- \\
3.512 \\
{[0.00} \\
7]\end{array}$ & $\begin{array}{c}9.044 \\
{[0.00} \\
0]\end{array}$ & $\begin{array}{c}- \\
3.094 \\
{[0.02} \\
7]\end{array}$ & $\begin{array}{c}4.947 \\
{[0.00} \\
0]\end{array}$ & $\begin{array}{c}- \\
5.656 \\
{[0.00} \\
0]\end{array}$ & \begin{tabular}{|c|}
- \\
6.369 \\
{$[0.00$} \\
$0]$
\end{tabular} & $\begin{array}{c}- \\
3.104 \\
{[0.02} \\
6]\end{array}$ & \begin{tabular}{|c}
- \\
9.170 \\
{$[0.00$} \\
$0]$
\end{tabular} & \begin{tabular}{|c|}
- \\
3.451 \\
{$[0.00$} \\
$9]$
\end{tabular} & $\begin{array}{c}- \\
8.861 \\
{[0.00} \\
0]\end{array}$ & $\begin{array}{c}4.352 \\
{[0.00} \\
0]\end{array}$ & $\begin{array}{c}- \\
7.760 \\
{[0.00} \\
0]\end{array}$ \\
\hline & $\begin{array}{c}\text { Con Iy } \\
T\end{array}$ & $\begin{array}{c}3.599 \\
{[0.03} \\
0]\end{array}$ & $\begin{array}{c}5.435 \\
{[0.00} \\
0]\end{array}$ & $\begin{array}{c}5.221 \\
{[0.00} \\
0]\end{array}$ & $\begin{array}{c}11.23 \\
3 \\
{[0.00} \\
0]\end{array}$ & $\begin{array}{c}3.423 \\
{[0.04} \\
8]\end{array}$ & $\begin{array}{c}5.229 \\
{[0.00} \\
0]\end{array}$ & $\begin{array}{c}5.657 \\
{[0.00} \\
0]\end{array}$ & $\begin{array}{c}6.366 \\
{[0.00} \\
0]\end{array}$ & $\begin{array}{c}3.325 \\
{[0.06} \\
2]\end{array}$ & $\begin{array}{c}9.314 \\
{[0.00} \\
0]\end{array}$ & $\begin{array}{c}3.853 \\
{[0.01} \\
4]\end{array}$ & $\begin{array}{c}9.215 \\
{[0.00} \\
0]\end{array}$ & $\begin{array}{c}4.728 \\
{[0.00} \\
0]\end{array}$ & $\begin{array}{c}7.948 \\
{[0.00} \\
0]\end{array}$ \\
\hline \multirow{3}{*}{$d m 1$} & $\begin{array}{c}\operatorname{Sin} I y \\
T\end{array}$ & 3.994 & $\begin{array}{c}- \\
10.11 \\
7\end{array}$ & 6.019 & 7.729 & 3.770 & 6.931 & 8.685 & 8.685 & $\begin{array}{c}- \\
1.733\end{array}$ & $\begin{array}{c}- \\
7.260\end{array}$ & 2.666 & $\begin{array}{c}- \\
5.314\end{array}$ & 3.383 & 7.437 \\
\hline & Con I & $\begin{array}{c}4.257 \\
{[0.00} \\
0]\end{array}$ & $\begin{array}{c}- \\
10.39 \\
8 \\
{[0.00} \\
0]\end{array}$ & $\begin{array}{c}6.553 \\
{[0.00} \\
0]\end{array}$ & $\begin{array}{c}8.184 \\
{[0.00} \\
0]\end{array}$ & $\begin{array}{c}3.913 \\
{[0.00} \\
1]\end{array}$ & $\begin{array}{c}7.014 \\
{[0.00} \\
0]\end{array}$ & $\begin{array}{c}10.03 \\
7 \\
{[0.00} \\
0]\end{array}$ & $\begin{array}{c}10.03 \\
7 \\
{[0.00} \\
0]\end{array}$ & $\begin{array}{c}3.782 \\
{[0.00} \\
3]\end{array}$ & $\begin{array}{c}9.011 \\
{[0.00} \\
0]\end{array}$ & $\begin{array}{c}3.591 \\
{[0.00} \\
5]\end{array}$ & $\begin{array}{c}- \\
6.501 \\
{[0.00} \\
0]\end{array}$ & $\begin{array}{c}3.413 \\
{[0.01} \\
0]\end{array}$ & $\begin{array}{c}7.473 \\
{[0.00} \\
0]\end{array}$ \\
\hline & $\begin{array}{c}\text { Con Iy } \\
T\end{array}$ & $\begin{array}{c}4.275 \\
{[0.00} \\
3]\end{array}$ & $\begin{array}{c}10.41 \\
3 \\
{[0.00} \\
0]\end{array}$ & $\begin{array}{c}6.586 \\
{[0.00} \\
0]\end{array}$ & $\begin{array}{c}8.234 \\
{[0.00} \\
0]\end{array}$ & $\begin{array}{c}- \\
3.730 \\
{[0.02} \\
0]\end{array}$ & $\begin{array}{c}6.978 \\
{[0.00} \\
0]\end{array}$ & $\begin{array}{c}9.956 \\
{[0.00} \\
0]\end{array}$ & $\begin{array}{c}9.956 \\
{[0.00} \\
0]\end{array}$ & $\begin{array}{c}3.700 \\
{[0.02} \\
2]\end{array}$ & $\begin{array}{c}8.953 \\
{[0.00} \\
0]\end{array}$ & $\begin{array}{c}3.497 \\
{[0.03} \\
9]\end{array}$ & $\begin{array}{c}- \\
6.515 \\
{[0.00} \\
0]\end{array}$ & $\begin{array}{c}4.043 \\
{[0.00} \\
7]\end{array}$ & $\begin{array}{c}8.038 \\
{[0.00} \\
0]\end{array}$ \\
\hline
\end{tabular}


REMEF (The Mexican Journal of Economics and Finance)

La pandemia Covid-19, la crisis financiera y la dinámica (Overshooting) del tipo de cambio

\begin{tabular}{|c|c|c|c|c|c|c|c|c|c|c|c|c|c|c|c|}
\hline \multirow{3}{*}{$d p i b$} & $\begin{array}{c}\operatorname{Sin} I y \\
T\end{array}$ & $\begin{array}{c}- \\
5.753\end{array}$ & $\begin{array}{c}11.26 \\
4\end{array}$ & 3.977 & $\begin{array}{c}- \\
4.829\end{array}$ & $\begin{array}{c}- \\
7.958\end{array}$ & $\begin{array}{c}- \\
7.958\end{array}$ & 2.093 & $\begin{array}{c}11.84 \\
1\end{array}$ & $\begin{array}{c}- \\
5.197\end{array}$ & $\begin{array}{c}- \\
9.507\end{array}$ & $\begin{array}{c}- \\
4.699\end{array}$ & $\begin{array}{c}- \\
9.730\end{array}$ & 4.000 & $\begin{array}{c}- \\
9.455\end{array}$ \\
\hline & Con I & $\begin{array}{c}- \\
6.222 \\
{[0.00} \\
0]\end{array}$ & $\begin{array}{c}- \\
11.84 \\
3 \\
{[0.00} \\
0]\end{array}$ & $\begin{array}{c}- \\
5.941 \\
{[0.00} \\
0]\end{array}$ & $\begin{array}{c}- \\
7.175 \\
{[0.00} \\
0]\end{array}$ & $\begin{array}{c}- \\
8.598 \\
{[0.00} \\
0]\end{array}$ & $\begin{array}{c}- \\
8.598 \\
{[0.00} \\
0]\end{array}$ & $\begin{array}{c}- \\
1.829 \\
{[0.36} \\
6]\end{array}$ & $\begin{array}{c}- \\
11.89 \\
9 \\
{[0.00} \\
0]\end{array}$ & $\begin{array}{c}- \\
5.391 \\
{[0.00} \\
0]\end{array}$ & $\begin{array}{c}- \\
9.716 \\
{[0.00} \\
0]\end{array}$ & $\begin{array}{c}- \\
4.801 \\
{[0.00} \\
0]\end{array}$ & $\begin{array}{c}- \\
9.744 \\
{[0.00} \\
0]\end{array}$ & $\begin{array}{c}- \\
4.789 \\
{[0.00} \\
0]\end{array}$ & $\begin{array}{c}- \\
10.66 \\
2 \\
{[0.00} \\
0]\end{array}$ \\
\hline & $\begin{array}{c}\text { Con Iy } \\
T\end{array}$ & $\begin{array}{c}- \\
6.411 \\
{[0.00} \\
0]\end{array}$ & $\begin{array}{c}- \\
11.99 \\
1 \\
{[0.00} \\
0]\end{array}$ & $\begin{array}{c}- \\
6.673 \\
{[0.00} \\
0]\end{array}$ & $\begin{array}{c}- \\
7.939 \\
{[0.00} \\
0]\end{array}$ & $\begin{array}{c}- \\
9.122 \\
{[0.00} \\
0]\end{array}$ & $\begin{array}{c}- \\
9.122 \\
{[0.00} \\
0]\end{array}$ & $\begin{array}{c}- \\
2.193 \\
{[0.49} \\
3]\end{array}$ & $\begin{array}{c}- \\
12.20 \\
3 \\
{[0.00} \\
0]\end{array}$ & $\begin{array}{c}- \\
5.410 \\
{[0.00} \\
0]\end{array}$ & $\begin{array}{c}- \\
9.660 \\
{[0.00} \\
0]\end{array}$ & $\begin{array}{c}- \\
4.926 \\
{[0.00} \\
0]\end{array}$ & $\begin{array}{c}- \\
9.819 \\
{[0.00} \\
0]\end{array}$ & $\begin{array}{c}- \\
4.903 \\
{[0.00} \\
0]\end{array}$ & $\begin{array}{c}- \\
10.68 \\
9 \\
{[0.00} \\
0]\end{array}$ \\
\hline \multirow{3}{*}{$\begin{array}{c}\text { Intius } \\
\quad a\end{array}$} & $\begin{array}{c}\operatorname{Sin} I y \\
T\end{array}$ & $\begin{array}{c}- \\
3.911\end{array}$ & $\begin{array}{c}- \\
6.974\end{array}$ & $\begin{array}{c}- \\
3.911\end{array}$ & $\begin{array}{c}- \\
6.974\end{array}$ & $\begin{array}{c}- \\
3.911\end{array}$ & $\begin{array}{c}- \\
6.974\end{array}$ & $\begin{array}{c}- \\
3.911\end{array}$ & $\begin{array}{c}- \\
6.974\end{array}$ & $\begin{array}{c}- \\
3.911\end{array}$ & $\begin{array}{c}- \\
6.974\end{array}$ & $\begin{array}{c}- \\
3.911\end{array}$ & $\begin{array}{c}- \\
6.974\end{array}$ & $\begin{array}{c}- \\
4.814\end{array}$ & $\begin{array}{c}- \\
6.913\end{array}$ \\
\hline & Con I & $\begin{array}{c}- \\
3.947 \\
{[0.00} \\
1]\end{array}$ & $\begin{array}{c}- \\
6.982 \\
{[0.00} \\
0]\end{array}$ & $\begin{array}{c}- \\
3.947 \\
{[0.00} \\
1]\end{array}$ & $\begin{array}{c}- \\
6.982 \\
{[0.00} \\
0]\end{array}$ & $\begin{array}{c}- \\
3.947 \\
{[0.00} \\
1]\end{array}$ & $\begin{array}{c}- \\
6.982 \\
{[0.00} \\
0]\end{array}$ & $\begin{array}{c}- \\
3.947 \\
{[0.00} \\
1]\end{array}$ & $\begin{array}{c}- \\
6.982 \\
{[0.00} \\
0]\end{array}$ & $\begin{array}{c}- \\
3.947 \\
{[0.00} \\
1]\end{array}$ & $\begin{array}{c}- \\
6.982 \\
{[0.00} \\
0]\end{array}$ & $\begin{array}{c}- \\
3.947 \\
{[0.00} \\
1]\end{array}$ & $\begin{array}{c}- \\
6.982 \\
{[0.00} \\
0]\end{array}$ & $\begin{array}{c}- \\
5.363 \\
{[0.00} \\
0]\end{array}$ & $\begin{array}{c}- \\
7.131 \\
{[0.00} \\
0]\end{array}$ \\
\hline & $\begin{array}{c}\text { Con Iy } \\
T\end{array}$ & $\begin{array}{c}- \\
3.960 \\
{[0.01} \\
0]\end{array}$ & $\begin{array}{c}- \\
6.942 \\
{[0.00} \\
0]\end{array}$ & $\begin{array}{c}- \\
3.960 \\
{[0.01} \\
0]\end{array}$ & $\begin{array}{c}- \\
6.942 \\
{[0.00} \\
0]\end{array}$ & $\begin{array}{c}- \\
3.960 \\
{[0.01} \\
0]\end{array}$ & $\begin{array}{c}- \\
6.942 \\
{[0.00} \\
0]\end{array}$ & $\begin{array}{c}- \\
3.960 \\
{[0.01} \\
0]\end{array}$ & $\begin{array}{c}- \\
6.942 \\
{[0.00} \\
0]\end{array}$ & $\begin{array}{c}- \\
3.960 \\
{[0.01} \\
0]\end{array}$ & $\begin{array}{c}6.942 \\
{[0.00} \\
0]\end{array}$ & $\begin{array}{c}3.960 \\
{[0.01} \\
0]\end{array}$ & $\begin{array}{c}6.942 \\
{[0.00} \\
0]\end{array}$ & $\begin{array}{c}- \\
5.475 \\
{[0.00} \\
0]\end{array}$ & $\begin{array}{c}- \\
7.108 \\
{[0.00} \\
0]\end{array}$ \\
\hline \multirow{3}{*}{ dlibor } & $\begin{array}{c}\operatorname{Sin} I y \\
T\end{array}$ & $\begin{array}{c}- \\
4.682\end{array}$ & $\begin{array}{c}- \\
4.951\end{array}$ & $\begin{array}{c}- \\
4.682\end{array}$ & $\begin{array}{c}- \\
4.951\end{array}$ & $\begin{array}{c}- \\
4.682\end{array}$ & $\begin{array}{c}- \\
4.951\end{array}$ & $\begin{array}{c}- \\
4.814\end{array}$ & $\begin{array}{c}- \\
6.913\end{array}$ & $\begin{array}{c}- \\
4.682\end{array}$ & $\begin{array}{c}- \\
4.951\end{array}$ & $\begin{array}{c}- \\
4.682\end{array}$ & $\begin{array}{c}- \\
4.951\end{array}$ & $\begin{array}{c}- \\
4.682\end{array}$ & $\begin{array}{c}- \\
4.951\end{array}$ \\
\hline & Con I & $\begin{array}{c}- \\
4.823 \\
{[0.00} \\
0]\end{array}$ & $\begin{array}{c}- \\
5.068 \\
{[0.00} \\
0]\end{array}$ & $\begin{array}{c}- \\
4.823 \\
{[0.00} \\
0]\end{array}$ & $\begin{array}{c}- \\
5.068 \\
{[0.00} \\
0]\end{array}$ & $\begin{array}{c}- \\
4.823 \\
{[0.00} \\
0]\end{array}$ & $\begin{array}{c}- \\
5.068 \\
{[0.00} \\
0]\end{array}$ & $\begin{array}{c}5.363 \\
{[0.00} \\
0]\end{array}$ & $\begin{array}{c}7.131 \\
{[0.00} \\
0]\end{array}$ & $\begin{array}{c}4.823 \\
{[0.00} \\
0]\end{array}$ & $\begin{array}{c}5.068 \\
{[0.00} \\
0]\end{array}$ & $\begin{array}{c}4.823 \\
{[0.00} \\
0]\end{array}$ & $\begin{array}{c}5.068 \\
{[0.00} \\
0]\end{array}$ & $\begin{array}{c}- \\
4.823 \\
{[0.00} \\
0]\end{array}$ & $\begin{array}{c}5.068 \\
{[0.00} \\
0]\end{array}$ \\
\hline & $\begin{array}{c}\text { Con } I y \\
T\end{array}$ & $\begin{array}{c}- \\
4.809 \\
{[0.00} \\
0]\end{array}$ & $\begin{array}{c}- \\
5.048 \\
{[0.00} \\
0]\end{array}$ & $\begin{array}{c}- \\
4.809 \\
{[0.00} \\
0]\end{array}$ & $\begin{array}{c}- \\
5.048 \\
{[0.00} \\
0]\end{array}$ & $\begin{array}{c}- \\
4.809 \\
{[0.00} \\
0]\end{array}$ & $\begin{array}{c}- \\
5.048 \\
{[0.00} \\
0]\end{array}$ & $\begin{array}{c}- \\
5.475 \\
{[0.00} \\
0]\end{array}$ & $\begin{array}{c}- \\
7.108 \\
{[0.00} \\
0]\end{array}$ & $\begin{array}{c}- \\
4.809 \\
{[0.00} \\
0]\end{array}$ & $\begin{array}{c}- \\
5.048 \\
{[0.00} \\
0]\end{array}$ & $\begin{array}{c}- \\
4.809 \\
{[0.00} \\
0]\end{array}$ & $\begin{array}{c}- \\
5.048 \\
{[0.00} \\
0]\end{array}$ & $\begin{array}{c}- \\
4.809 \\
{[0.00} \\
0]\end{array}$ & $\begin{array}{c}- \\
5.048 \\
{[0.00} \\
0]\end{array}$ \\
\hline
\end{tabular}

Fuente: Elaboración de los autores.

1/ Las tasas de interés foráneas para Reino Unido son las prevalecientes en Estados Unidos y la Zona Euro; 2/ Para aproximar la tasa de interés extranjera empleamos el indicador LIBOR y el de la eurozona. Valor-p de los parámetros entre corchetes. Nota 1. MEX: México; KOR: Corea del Sur; BRA: Brasil; UK: Reino Unido; DEU: Alemania; JPN: Japón; USA: Estados Unidos. Nota 2. tcn: tipo de cambio nominal; tid: tasa de interés; ipc: índice de precios al consumidor; m1: agregado monetario M1; pib: producto interno bruto; tiusa: tasa de interés de Estados Unidos; libor: tasa de interés LIBOR.

\section{Cuadro A2. Prueba de Pesaran, Shin y Smith}

Ho: No existe una relación de largo plazo

\begin{tabular}{|c|c|c|c|c|c|c|c|c|}
\hline \multirow{2}{*}{ País } & \multirow{2}{*}{ Especificación } & \multirow{2}{*}{ Estadístico } & \multicolumn{2}{|c|}{$10 \%$} & \multicolumn{2}{|c|}{$5 \%$} & \multicolumn{2}{c|}{$1 \%$} \\
\cline { 3 - 9 } & & & $I(0)$ & $I(1)$ & $I(0)$ & $I(1)$ & $I(0)$ & $I(1)$ \\
\hline \multirow{3}{*}{ México } & \multirow{2}{*}{ Modelo 1 } & $F=3.872$ & 2.130 & 3.166 & 2.490 & 3.622 & 3.294 & 4.627 \\
\cline { 3 - 10 } & & $t=-4.792$ & -2.514 & -3.812 & -2.839 & -4.185 & -3.480 & -4.906 \\
\cline { 3 - 9 } & \multirow{2}{*}{ Modelo 2 } & $F=8.210$ & 2.055 & 3.111 & 2.389 & 3.541 & 3.135 & 4.486 \\
\cline { 3 - 9 } & & $t=-5.644$ & -2.519 & -4.010 & -2.844 & -4.388 & -3.486 & -5.120 \\
\hline \multirow{2}{*}{ Corea del Sur } & \multirow{2}{*}{ Modelo 1 } & $F=6.708$ & 2.148 & 3.155 & 2.508 & 3.606 & 3.311 & 4.593 \\
\cline { 3 - 9 } & & $t=-4.244$ & -2.531 & -3.837 & -2.853 & -4.205 & -3.488 & -4.916 \\
\hline
\end{tabular}



DOI: https://doi.org/10.21919/remef.v16i3.671

\begin{tabular}{|c|c|c|c|c|c|c|c|c|}
\hline & Modolo 2 & $F=5.996$ & 2.060 & 3.107 & 2.395 & 3.536 & 3.140 & 4.475 \\
\hline & Mivueto 2 & $t=-4.026$ & -2.525 & -4.019 & -2.849 & -4.396 & -3.489 & -5.124 \\
\hline \multirow{4}{*}{ Brasil } & \multirow{2}{*}{ Modelo 1} & $F=2.116$ & 2.117 & 3.173 & 2.477 & 3.634 & 3.283 & 4.650 \\
\hline & & $t=-2.686$ & -2.502 & -3.795 & -2.829 & -4.172 & -3.474 & -4.899 \\
\hline & \multirow{2}{*}{ Modelo 2} & $F=4.243$ & 2.031 & 3.125 & 2.366 & 3.563 & 3.114 & 4.530 \\
\hline & & $t=-3.509$ & -2.495 & -3.974 & -2.824 & -4.358 & -3.474 & -5.103 \\
\hline \multirow{4}{*}{ Reino Unido } & \multirow{2}{*}{ Modelo 1} & $F=6.943$ & 2.146 & 3.208 & 2.519 & 3.688 & 3.362 & 4.757 \\
\hline & & $t=-3.459$ & -2.505 & -3.804 & -2.837 & -4.188 & -3.494 & -4.934 \\
\hline & \multirow{2}{*}{ Modelo 2} & $F=7.744$ & 2.074 & 3.154 & 2.421 & 3.607 & 3.206 & 4.616 \\
\hline & & $t=-4.414$ & -2.511 & -4.000 & -2.843 & -4.389 & -3.500 & -5.148 \\
\hline \multirow{4}{*}{ Alemania } & \multirow{2}{*}{ Modelo 1} & $F=3.285$ & 2.167 & 3.144 & 2.527 & 3.589 & 3.327 & 4.559 \\
\hline & & $t=-4.282$ & -2.549 & -3.862 & -2.868 & -4.226 & -3.496 & -4.926 \\
\hline & \multirow{2}{*}{ Modelo 2} & $F=3.440$ & 2.043 & 3.118 & 2.377 & 3.552 & 3.125 & 4.508 \\
\hline & & $t=-4.927$ & -2.507 & -3.992 & -2.834 & -4.373 & -3.480 & -5.112 \\
\hline \multirow{4}{*}{ Japón } & \multirow{2}{*}{ Modelo 1} & $F=3.547$ & 2.092 & 3.187 & 2.452 & 3.656 & 3.261 & 4.696 \\
\hline & & $t=-3.898$ & -2.478 & -3.762 & -2.810 & -4.145 & -3.463 & -4.885 \\
\hline & \multirow{2}{*}{ Modelo 2} & $F=4.376$ & 1.949 & 3.176 & 2.283 & 3.641 & 3.041 & 4.684 \\
\hline & & $t=-4.230$ & -2.411 & -3.846 & -2.755 & -4.254 & -3.432 & -5.046 \\
\hline \multirow{4}{*}{$U S A$} & \multirow{2}{*}{ Modelo 1} & $F=4.497$ & 2.154 & 3.151 & 2.515 & 3.600 & 3.316 & 4.581 \\
\hline & & $t=-3.267$ & -2.537 & -3.846 & -2.858 & -4.212 & -3.491 & -4.919 \\
\hline & \multirow{2}{*}{ Modelo 2} & $F=4.180$ & 2.099 & 3.134 & 2.446 & 3.576 & 3.225 & 4.555 \\
\hline & & $t=-3.938$ & -2.539 & -4.040 & -2.865 & -4.421 & -3.512 & -5.163 \\
\hline
\end{tabular}

Fuente: Elaboración de los autores

Valor-p entre corchetes

\section{Cuadro A.3. Prueba LM (estadístico de Breusch-Godfrey)}

Ho: No hay presencia de autocorrelación

\begin{tabular}{|c|c|c|c|c|c|c|}
\hline \multirow{2}{*}{ País } & \multirow{2}{*}{ Especificación } & \multicolumn{5}{|c|}{ Rezago (p) } \\
\hline & & 1 & 2 & 3 & 4 & 5 \\
\hline \multirow{3}{*}{ México } & Modelo 1 & 0.052 & 0.066 & 0.044 & 0.043 & 0.129 \\
\hline & & [0.8208] & [0.9364] & [0.9877] & [0.9965] & [0.9854] \\
\hline & Modelo 2 & $\begin{array}{c}2.680 \\
{[0.1062]}\end{array}$ & $\begin{array}{c}1.581 \\
{[0.2134]}\end{array}$ & $\begin{array}{c}1.284 \\
{[0.2872]}\end{array}$ & $\begin{array}{c}1.068 \\
{[0.3797]}\end{array}$ & $\begin{array}{c}0.901 \\
{[0.4858]}\end{array}$ \\
\hline \multirow{2}{*}{ Corea del Sur } & Modelo 1 & $\begin{array}{c}0.037 \\
{[0.8474]}\end{array}$ & $\begin{array}{c}0.527 \\
{[0.5927]}\end{array}$ & $\begin{array}{c}0.370 \\
{[0.7748]}\end{array}$ & $\begin{array}{c}0.499 \\
{[0.7365]}\end{array}$ & $\begin{array}{c}0.400 \\
{[0.8473]}\end{array}$ \\
\hline & Modelo 2 & $\begin{array}{c}0.116 \\
{[0.7339]}\end{array}$ & $\begin{array}{c}0.858 \\
{[0.4286]}\end{array}$ & $\begin{array}{c}0.667 \\
{[0.5751]}\end{array}$ & $\begin{array}{c}0.822 \\
{[0.5160]}\end{array}$ & $\begin{array}{c}0.658 \\
{[0.6566]}\end{array}$ \\
\hline \multirow{2}{*}{ Brasil } & Modelo 1 & $\begin{array}{c}0.706 \\
{[0.4038]}\end{array}$ & $\begin{array}{c}2.650 \\
{[0.0784]}\end{array}$ & $\begin{array}{c}1.783 \\
{[0.1594]}\end{array}$ & $\begin{array}{c}1.649 \\
{[0.1734]}\end{array}$ & $\begin{array}{c}1.319 \\
{[0.2680]}\end{array}$ \\
\hline & Modelo 2 & $\begin{array}{c}0.007 \\
{[0.9335]}\end{array}$ & $\begin{array}{c}0.157 \\
{[0.8550]}\end{array}$ & $\begin{array}{c}0.431 \\
{[0.7314]}\end{array}$ & $\begin{array}{c}0.805 \\
{[0.5269]}\end{array}$ & $\begin{array}{c}0.661 \\
{[0.6547]}\end{array}$ \\
\hline \multirow{2}{*}{ Reino Unido } & Modelo 1 & $\begin{array}{c}2.708 \\
{[0.1055]}\end{array}$ & $\begin{array}{c}2.192 \\
{[0.1216]}\end{array}$ & $\begin{array}{c}1.600 \\
{[0.2004]}\end{array}$ & $\begin{array}{c}1.506 \\
{[0.2141]}\end{array}$ & $\begin{array}{c}1.693 \\
{[0.1532]}\end{array}$ \\
\hline & Modelo 2 & $\begin{array}{c}0.126 \\
{[0.7235]}\end{array}$ & $\begin{array}{c}0.064 \\
{[0.9382]}\end{array}$ & $\begin{array}{c}0.082 \\
{[0.9696]}\end{array}$ & $\begin{array}{c}0.232 \\
{[0.9190]}\end{array}$ & $\begin{array}{c}0.438 \\
{[0.8202]}\end{array}$ \\
\hline Alemania & Modelo 1 & $\begin{array}{c}0.261 \\
{[0.6109]}\end{array}$ & $\begin{array}{c}0.336 \\
{[0.7158]}\end{array}$ & $\begin{array}{c}1.032 \\
{[0.3839]}\end{array}$ & $\begin{array}{c}0.923 \\
{[0.4556]}\end{array}$ & $\begin{array}{c}0.739 \\
{[0.5969]}\end{array}$ \\
\hline
\end{tabular}


REMEF (The Mexican Journal of Economics and Finance)

La pandemia Covid-19, la crisis financiera y la dinámica (Overshooting) del tipo de cambio

\begin{tabular}{|c|c|c|c|c|c|c|}
\hline & Modelo 2 & $\begin{array}{c}0.214 \\
{[0.6453]}\end{array}$ & $\begin{array}{c}1.498 \\
{[0.2313]}\end{array}$ & $\begin{array}{c}1.357 \\
{[0.2640]}\end{array}$ & $\begin{array}{c}1.122 \\
{[0.3540]}\end{array}$ & $\begin{array}{c}0.947 \\
{[0.4570]}\end{array}$ \\
\hline \multirow{4}{*}{ Japón } & \multirow{2}{*}{ Modelo 1 } & 0.228 & 1.719 & 1.357 & 1.296 & 1.903 \\
& & {$[0.6351]$} & {$[0.1879]$} & {$[0.2649]$} & {$[0.2823]$} & {$[0.1080]$} \\
\cline { 2 - 7 } & \multirow{2}{*}{ Modelo 2 } & 0.378 & 0.191 & 3.651 & 3.126 & 3.168 \\
& & {$[0.5414]$} & {$[0.8271]$} & {$[0.0189]$} & {$[0.0232]$} & {$[0.0154]$} \\
\hline \multirow{3}{*}{$\begin{array}{c}\text { Estados } \\
\text { Unidos }\end{array}$} & \multirow{2}{*}{ Modelo 1 } & 1.223 & 2.213 & 1.487 & 1.863 & 1.674 \\
\cline { 2 - 7 } & \multirow{2}{*}{ Modelo 2 } & {$[0.2726]$} & {$[0.1170]$} & {$[0.2258]$} & {$[0.1270]$} & {$[0.1528]$} \\
\hline & & {$[0.2320]$} & {$[0.063$} & 3.621 & 3.094 & 3.047 \\
& & {$[0.0182]$} & {$[0.0225]$} & {$[0.0168]$} \\
\hline
\end{tabular}

Fuente: Elaboración de los autores

Valor-p estimados entre corchetes

\section{Cuadro A.4. Prueba de heteroscedasticidad (estadístico de White)}

Ho: Los residuos son homocedásticos

\begin{tabular}{|c|c|c|c|c|c|c|c|}
\hline Especificación & México & Corea & Brasil & $\begin{array}{l}\text { Reino } \\
\text { Unido }\end{array}$ & Alemania & Japón & $U S A$ \\
\hline Modelo 1 & $\begin{array}{c}83.00 \\
{[0.4484]}\end{array}$ & $\begin{array}{c}16.39 \\
{[0.9452]}\end{array}$ & $\begin{array}{c}83.00 \\
{[0.4484]}\end{array}$ & $\begin{array}{c}71.00 \\
{[0.4442]}\end{array}$ & $\begin{array}{c}62.28 \\
{[0.0361]}\end{array}$ & $\begin{array}{c}83.00 \\
{[0.4484]}\end{array}$ & $\begin{array}{c}66.43 \\
{[0.4273]}\end{array}$ \\
\hline Modelo 2 & $\begin{array}{c}83.00 \\
{[0.4484]}\end{array}$ & $\begin{array}{c}23.73 \\
{[0.8821]}\end{array}$ & $\begin{array}{c}83.00 \\
{[0.4484]}\end{array}$ & $\begin{array}{c}71.00 \\
{[0.4442]}\end{array}$ & $\begin{array}{c}83.00 \\
{[0.4484]}\end{array}$ & $\begin{array}{c}0.19 \\
{[0.6605]}\end{array}$ & $\begin{array}{c}57.53 \\
{[0.3460]}\end{array}$ \\
\hline
\end{tabular}

Fuente: Elaboración de los autores

Valor-p estimados entre corchetes

\section{Cuadro A.5. Prueba de Normalidad (estadístico de Royston)}

Ho: Los errores se distribuyen normalmente

\begin{tabular}{|c|c|c|c|c|c|c|c|}
\hline Estadístico & México & Corea & Brasil & $\begin{array}{c}\text { Reino } \\
\text { Unido }\end{array}$ & Alemania & Japón & USA \\
\hline \multirow{2}{*}{ Royston } & $\begin{array}{c}39.47 \\
{[0.0000]}\end{array}$ & $\begin{array}{c}30.98 \\
{[0.0000]}\end{array}$ & $\begin{array}{c}63.55 \\
{[0.0000]}\end{array}$ & $\begin{array}{c}15.33 \\
{[0.0005]}\end{array}$ & $\begin{array}{c}4.49 \\
{[0.1060]}\end{array}$ & $\begin{array}{c}79.07 \\
{[0.0000]}\end{array}$ & $\begin{array}{c}17.97 \\
{[0.0001]}\end{array}$ \\
\hline \multirow{5}{*}{} & 47.35 & 29.64 & 48.40 & 16.19 & 4.34 & 75.45 & 2.90 \\
& {$[0.0000]$} & {$[0.0000]$} & {$[0.0000]$} & {$[0.0003]$} & {$[0.1144]$} & {$[0.0000]$} & {$[0.2350]$} \\
\hline
\end{tabular}

Fuente: Elaboración de los autores

Valor-p estimados entre corchetes 